\title{
ON SOLVABLE GROUPS OF FINITE MORLEY RANK
}

\author{
ALI NESIN
}

\begin{abstract}
We investigate solvable groups of finite Morley rank. We find conditions on $G$ for $G^{\prime}$ to split in $G$. In particular, if $G^{\prime}$ is abelian and $Z(G)=1$ we prove that $G=G^{\prime} \rtimes T$ for some $T$ and the $\operatorname{ring} \mathbf{Z}[T] /$ ann $G^{\prime}$ is intepretable in $G$. We exploit the methods used in proving these results to find more information about solvable groups.
\end{abstract}

\section{INTRODUCTION}

The concept of Morley rank was introduced by Morley in 1965 [Mo]. Roughly speaking, in a structure of finite Morley rank every definable subset of endowed with two integers: the Morley rank and the Morley degree. The first number behaves like the dimension and the second one like the number of irreducible components of constructible sets of algebraic geometry (see, e.g., [Sp] for the definition of a constructible set). [ $\mathrm{La}, \mathrm{Pi}, \mathrm{Po} 1, \mathrm{Po} 2]$ give a detailed treatment of structures of finite Morley rank. In [Th1] and [ $\mathrm{Nel}$ ], the reader may find a faster introduction to the subject as far as groups are concerned.

In the 1970s, Cherlin and Zil'ber independently discovered that groups of finite Morley rank behave like geometric groups and in view of Macintyre's result (infinite fields of finite Morley rank are algebraically closed) they made the following conjecture:

Conjecture. Infinite simple groups of finite Morley rank are algebraic groups over algebraically closed fields.

We are still far away from proving this conjecture. But considerable progress has been made in the last few years. First of all, Thomas proved the conjecture for locally finite groups using the classification of finite simple groups [Th2]. Second, Zil'ber showed that these groups look very much like algebraic groups [Zi2]. In $\S 3$ we will give a partial list of his results.

In an algebraic group a Borel subgroup is a maximal (algebraically) connected solvable subgroup. Borel subgroups play a very important role in the study of algebraic groups. Similarly, one may define a Borel subgroup of a group of finite Morley rank as a maximal (model theotically) connected solvable subgroup and

Received by the editors May 4, 1988 and, in revised form, November 8, 1988.

1980 Mathematics Subject Classification (1985 Revision). Primary 03C60; Secondary 03C45, $20 \mathrm{~F} 16$. 
expect that one day they will be important in solving the above conjecture. We can justify our faith by saying that we know more about solvable groups of finite Morley rank than about simple groups (unless they are nilpotent, but Borels are supposed to be centerless). In this article we investigate these solvable groups and in the particular case where the group is solvable of class 2 we show strong resemblances with algebraic groups.

This paper may be seen as a sequel to [Ne2]. But it is self-contained.

In $\S 2$ we fix our group theoretical notation. We also state some basic theorems to refresh the model theorist's memory.

In $\S 3$ we do the same for model theory of groups. In that section we state some known facts that will be used in later sections.

In $\S 4$ we define the socle of a solvable group and investigate its basic properties; in particular, we look closely at the action of the group on its socle. This action was completely described in [Ne2]. We survey the results here.

In $\S 5$ we prepare the inductive step to prove two theorems and we prove them:

Theorem 1. If $G$ is a connected, solvable group of class 2 and of finite Morley rank and if $G^{\prime} \cap Z(G)=1$, then there is a definable subgroup $T$ of $G$ for which

$$
G=G^{\prime} \rtimes T \text {. }
$$

Theorem 2. If $G$ is a connected, solvable group of finite Morley rank and if for some $g \in G, C_{G^{\prime}}(g)=1$, then there is a definable subgroup $T$ of $G$ for which

$$
G=G^{\prime} \rtimes T .
$$

We give several examples to illustrate the theorems and Example 7 shows that the supplementary hypotheses of these two theorems $\left(G^{\prime} \cap Z(G)=1\right.$ and $\left.C_{G^{\prime}}(g)=1\right)$ are crucial.

In the next section we investigate in detail solvable groups of class 2 . We imbed each connected, centerless, solvable group of class 2 of finite Morley rank in another group with the same properties (Theorem 3). But this bigger group looks more like an algebraic group than the one we started with. We call it the full group of $G$ and denote it by $\widetilde{G}$. Then we look at $\widetilde{G}$ more attentively. We prove the following.

Theorem 4. $\widetilde{G}=\bigoplus_{i=1}^{\prime} G_{i}^{\prime} \rtimes R_{i}^{*}$ where $G^{\prime}=\bigoplus_{i=1}^{\prime} G_{i}^{\prime}$ and each $G_{i}^{\prime}$ is a definable normal subgroup of $G, R_{i}$ is a connected, local, Artinian and Noetherian ring with identity and of finite Morley rank.

There is a complete classification of these rings (see Cohen [Co], also [Sa]).

The next theorem gives a smaller imbedding of $G$.

In $\S 7$, we define the Fitting subgroup of a group of finite Morley rank as being the maximal connected nilpotent normal subgroup. The main result of this section is the following theorem.

Theorem 6. Let $G$ be a solvable connected group of finite Morley rank. If $F$ is the Fitting subgroup of $G$, then $G / F$ is a divisible group. 
In $\S 8$ we look at the Fitting subgroup $F$ of a centerless, connected solvable algebraic group. We show that the unipotent subgroup $U$ is definable (Theorem 7) and if the language is the pure language of groups then $U=F$. This result is proved with Anand Pillay.

In the final section we show that if $G$ is a connected, centerless, solvable group of finite Morley rank whose definable connected proper subgroups are nilpotent, then $G=k \rtimes T$ where $T<k^{*}$ and $k$ is an algebraically closed field (Theorem 7). This generalizes Cherlin's classification of centerless groups of Morley rank 2 (see [Ch]). We hope this result will be helpful in proofs by induction.

\section{GROUP THEORETICAL AND ALGEBRAIC BACKGROUND}

$G$ will always stand for a group. We will denote its identity element by 1. $Z(G)$ stands for its center. If $H$ is a subgroup of $G$ we write $H<G$. If $H$ is normal in $G$ then we denote this fact by $H \triangleleft G$. If $x, h \in G$ then $x^{h}=h^{-1} x h$. Clearly $\left(x^{h}\right)^{g}=x^{h g}$. If $n \in \mathbf{N}, x^{n h}$ means $\left(x^{n}\right)^{h}$ or $\left(x^{h}\right)^{n}$. If $X \subseteq G, H<G$ and if for all $x \in X, h \in H, x^{h} \in X$, then we will say that $X$ is $H$-normal. $[x, h]$ stands for $x^{-1} h^{-1} x h$. If $X$ is an abelian subgroup which is normalized by $H$ then $[x y, h]=[x, h][y, h]$ for all $x, y \in X$, all $h \in H$. [X,H] denotes the subgroup of $G$ generated by the set $\{[x, h]: x \in X, h \in H\}$. The subgroups $G^{i}, G^{(i)}$ are defined inductively.

$$
\begin{aligned}
G^{0} & =G^{(0)}=G, \\
G^{i+1} & =\left[G, G^{i}\right], \\
G^{(i+1)} & =\left[G^{(i)}, G^{(i)}\right] .
\end{aligned}
$$

These subgroups are normal. We have $G^{1}=G^{(1)}=[G, G]$. We denote it by $G^{\prime}$. $G^{\prime}$ is called the derived subgroup of $G$. It is the smallest subgroup $H$ of $G$ for which $G / H$ is abelian. Any subgroup of $G$ that contains $G^{\prime}$ is normal. We have $G^{i+1} \subseteq G^{i}$ and $G^{(i+1)} \subseteq G^{(i)}$. A group $G$ is said to be solvable if $G^{(i)}=1$ for some $i$. If $i$ happens to be the least integer for which $G^{(i)}=1$, then $G$ is called solvable of class $i$. If $G$ is solvable then $G \neq G^{\prime}$. A group $G$ is said to be nilpotent if $G^{i}=1$ for some $i$. Define the $i$ th center of $G$, $Z_{i}(G)$, by induction on $i$.

$$
\begin{aligned}
Z_{0} & =Z_{0}(G)=\{1\}, \\
Z_{i+1} & =Z_{i+1}(G)=\left\{g \in G:[G, g] \subseteq Z_{i}(G)\right\} .
\end{aligned}
$$

Then $Z_{i}(G) \triangleleft G, Z_{i} \subseteq Z_{i+1}$, and

$$
Z_{i+1} / Z_{i}=Z\left(G / Z_{i}\right)
$$

$G$ is nilpotent iff $Z_{i}(G)=G$ for some $i$. Thus a nilpotent group has nontrivial center. Every nontrivial normal subgroup of a nilpotent group intersects the center nontrivially. Subgroups and quotients of nilpotent (resp. solvable) groups 
are nilpotent (resp. solvable). If $H$ and $G / H$ are solvable then so is $G$ (here $H \triangleleft G)$. Upper triangular matrices with nonzero determinant form a solvable group which is not nilpotent. Strictly upper-triangular matrices (1's on the diagonal) form a nilpotent group.

If $A \triangleleft H$ are subgroups of a group $G$, then $(H / A)^{\prime}=H^{\prime} A / A$.

If $X, Y \subseteq G$ then we denote by $X Y$ the subset $X Y=\{x y: x \in X, y \in Y\}$.

If $\left(X_{i}\right)_{i \in I}$ are subsets of $G,\left\langle X_{i} \mid i \in I\right\rangle$ denotes the group generated by all the $X_{i}$ 's. It is the smallest subgroup of $G$ containing $\bigcup_{i} X_{i}$. If $I$ is a finite set $\{1, \ldots, n\}$, we write $\left\langle X_{1}, \ldots, X_{n}\right\rangle$. If $H \triangleleft G, K<G$, then $\langle H, K\rangle=\{h k \mid h \in H, k \in K\}$. We denote it by $H K$. If $K \triangleleft G$ also (and $H$ and $K$ are nilpotent) then $H K \triangleleft G$ (and $H K$ is nilpotent).

We say that a group $G$ is the semidirect product of two subgroups $H$ and $K$ if

(i) $H \triangleleft G$,

(ii) $G=H K$,

(iii) $H \cap K=\{1\}$.

In this case every element of $g$ can be written uniquely as $h k$, for $h \in H$, $k \in K$. We write $G=H \rtimes K$ if $G$ is the semidirect product of $H$ and $K$ (in that order). If $G=H \rtimes K$ then we have a homomorhism

$$
\varphi: K \rightarrow \text { Aut } H
$$

given by

$$
\varphi(k)(h)=h^{k^{-1}} .
$$

We say that a group $K$ acts on a group $H$ if a homomorphism

$$
\varphi: K \rightarrow \text { Aut } H
$$

is given. In that case we can construct a group $G$ which is isomorphic to $H \rtimes K$ as follows. We define

$$
G=H \times K
$$

as a set. The product is defined as follows:

$$
(h, k)\left(h^{\prime}, k^{\prime}\right)=\left(h \cdot \varphi(k) h^{\prime}, k k^{\prime}\right) .
$$

It is easy to check that $G$ is a group and it is the semidirect product of $H \times\{1\}$ and $\{1\} \times K$. The best way to visualize $G$ is as follows: think of $G$ as the set

$$
\left\{\left(\begin{array}{cc}
k & h \\
0 & 1
\end{array}\right): k \in K, h \in H\right\}
$$

and the multiplication as the matrix multiplication $\left(k h^{\prime}\right.$ meaning $\left.\varphi(k) h^{\prime}\right)$. If $H=k^{+}, K=k \backslash\{0\}$ where $k$ is a field, we obtain the affine group in this way. In this case the action is multiplication:

$$
\varphi(\lambda) a=\lambda a
$$

for $\lambda \in k^{*}, a \in k$. 
If $G=H \rtimes K$ for some subgroup $K$, we say that $H$ splits in $G$. If $H$ and $K$ are abelian then $G$ is solvable of class at most 2 .

If $G$ is a solvable, (algebraically) connected linear algebraic group then $G$ is conjugate to upper-triangular matrices (Lie-Kolchin theorem; see, e.g., [Sp], Chapter 6). Suppose $G$ is in upper-triangular form. Then the set of strictly upper-triangular matrices of $G$ is denoted by $U . U$ is called the unipotent subgroup (or radical) of $G$. It is clearly a normal subgroup of $G . U$ is nilpotent and it contains $G^{\prime} . U$ is a connected subgroup of $G$. The set of diagonalizable matrices of $G$ contains a closed connected subgroup $T$ which is maximal for these properties. It is called a maximal torus. One has

$$
G=U \rtimes T .
$$

If $H \subseteq K$ are subgroups of $G,[K: H]$ will denote the index of $H$ in $K$, i.e., $[K: H]=\operatorname{Card}(K / H)$.

If $H, K$ are subgroups of $G, C_{H}(K)$ will mean the centralizer of $K$ in $H$, i.e., $C_{H}(K)=\{h \in H: h k=k h$ for all $k \in K\} . C_{H}(K)$ is a subgroup of $G$. If $K$ and $H$ are normal in $G$ then so is $C_{H}(K)$.

If $R$ is a ring, $I$ an ideal of $R$, we write $I \triangleleft R . R^{*}$ stands for the set of invertible elements of $R$. It is a group under multiplication.

$\mathbf{Z}[G]$ denotes the group ring of $G$ over $\mathbf{Z}$. It is defined as the formal set

$$
\left\{\sum_{i=1}^{k} n_{i} g_{i}: k \in \mathbf{N}, \dot{n}_{i} \in \mathbf{Z}, g_{i} \in G\right\}
$$

with the obvious addition and multiplication. If $G$ is an Abelian group acting on another abelian group $A$, then we can extend this action to $\mathbf{Z}[G]$ by (denoting $A$ additively)

$$
\left(\sum n_{i} g_{i}\right)(a)=\sum n_{i} g_{i} a
$$

where $g_{i} a$ is the element of $A$ which is the result of the $g_{i}$ action on $a$. If $\lambda=\sum n_{i} g_{i}$, the length of $\lambda$ is $l(\lambda)=\sum\left|n_{i}\right|$.

Let $M$ be an $R$-module. Let $X \subseteq M$. Then the annihilator of $X$ in $R$ is

$$
\text { ann } X=\operatorname{ann}_{R} X=\{r \in R \mid r X=0\} .
$$

This is a left-ideal of $R$. If $X$ is a submodule then ann $X \triangleleft R$. Then $X$ becomes an $R /$ ann $X$-module in the obvious way.

An ideal $I$ of a ring $R$ is said to be nilpotent if for some integer $n, I^{n}=0$, i.e., if for any $x_{1}, \ldots, x_{n} \in I$ we have $x_{1} \cdots x_{n}=0$.

A commutative ring $R$ with identity is local if it has a unique maximal ideal $M$. Then $R \backslash M=R^{*}$ and $R / M$ is a field.

If $I \triangleleft R, I^{n}$ denotes the ideal generated by $x_{1} \cdots x_{n}$ for all $x_{1}, \ldots, x_{n} \in I$. Clearly

$$
I^{n}=\left\{\sum_{k=1}^{m} x_{k 1} \cdots x_{k n_{k}}: m \in \mathbf{N}, x_{i j} \in I\right\} \text {. }
$$


$\mathbf{Z}_{p^{\infty}}$ denotes the Prüfer group, i.e.,

$$
\mathbf{Z}_{p^{\infty}}=\left\{z \in \mathbf{C}: z^{p^{n}}=1 \text { for some } n\right\}
$$

\section{MODEL THEORY OF GROUPS. BACKGROUND}

If $M$ is a structure for a language $\mathscr{L}$, by a definable subset of $M$ we will mean a subset which is first-order definable using parameters from $M$.

Let $G$ be a group of finite Morley rank. So to each definable subset $X$ of $G$ we attached two integers: $r$ and $d . r$ will be called the (Morley) rank, $d$ the (Morley) degree of $X$. We will write $\operatorname{rk} X=r, \operatorname{deg} X=d$.

If $H \subseteq K$ are definable subsets of $G$ then $\operatorname{rk} H \leq \operatorname{rk} K$. If they have the same rank then $\operatorname{deg} H \leq \operatorname{deg} K$. If $H$ and $K$ are also subgroups and if rk $H=\operatorname{rk} K$, then $\operatorname{deg} K=[K: H] \operatorname{deg} H$. In particular, $K=H$ iff $\operatorname{rk} K=$ rk $H, \operatorname{deg} K=\operatorname{deg} H$. This shows that a group of finite Morley rank satisfies descending chain condition on definable subgroups. This has an important consequence: if $H$ is a definable subgroup then

$$
H^{\circ}=\bigcap\{K: K<G, K \text { definable, } K \subseteq H,[H: K]<\infty\}
$$

is the intersection of finitely many such subgroups, thus has finite index in $H$. $H^{\circ}$ is called the connected component of $H$. It is the smallest subgroup of $H$ which is definable and has finite index in $H$. In other words, $H^{\circ}$ has no definable proper subgroups of finite index, and it is the biggest such definable subgroup of $G$. If $H \triangleleft G$, then $H^{\circ} \triangleleft G$ also. Cherlin [Ch] proved that $\operatorname{deg} H$ is exactly $\left[H: H^{\circ}\right]$. Thus $\operatorname{deg} H^{\circ}=1 . H$ is connected iff $\operatorname{deg} H=1$, equivalently if $H=H^{\circ}$. The sentence " $H$ is connected" will always mean " $H$ is definable and connected."

If $K \triangleleft H$ are definable subgroups and if $K$ and $H / K$ are connected then $H$ is connected. If $H \triangleleft G$ is definable and infinite then $\operatorname{rk} G>\operatorname{rk} G / H$. Definable subgroups of $G / H$ (in the $G$-language) are of the form $K / H$ where $K$ is a definable subgroup of $G$ containing $H$.

We will now list some of the known facts that we will repeatedly use in the sequel. The proof of most of them can be found in [Po2]. Several of them are proved in more generality.

Fact 1 (Macintyre [B.Ch.Mac]). Let $G$ be a group of finite Morley rank. Let $H$ be a connected subgroup of $G$. Then any finite, $H$-normal subset of $G$ is in the centralizer of $H$.

Fact 2 (Zil'ber [Z2]). Let $A_{i}<G$ be a family of connected subgroups of a group $G$ of finite Morley rank. Then the subgroup

$$
H=\left\langle A_{i}: i \in I\right\rangle
$$

is connected. In fact, for some $i_{1}, \ldots, i_{n} \in I$

$$
H=A_{i_{1}} \cdots A_{i_{n}} .
$$


If each $A_{i}$ is Abelian and they commute with each other (i.e., if $H$ is Abelian), we may denote the multiplication in $H$ additively and write

$$
H=A_{i_{1}}+\cdots+A_{i_{n}} .
$$

Fact 3 ( $\mathrm{Zil}{ }^{\prime}$ ber $\left.[\mathrm{Zi} 2]\right)$. Let $G$ be a group of finite Morley rank. Let $H<G$ be a connected subgroup of $G$. If $X \subseteq G$ is any subset of $G$ then the subgroup $[H, X]$ is connected.

Fact 4 (Zil'ber [Zi2]). If $G$ is a connected group of finite Morley rank then $G^{i}$, $G^{(i)}$ are connected.

Fact 5 (Zil'ber [Zi1]/Nesin [Ne2]). Let $G$ be a connected solvable group of finite Morley rank. Then $G^{\prime}$ is nilpotent (and connected by Fact 4).

Fact 6 (Macintyre [Mac2]). An infinite field of finite Morley rank is an algebraically closed field. It is also connected.

Fact 7 (Macintrye [Mac1]). An Abelian group $G$ of finite Morley rank is of the form

$$
G=D \oplus B
$$

where $D$ is a divisible subgroup and $B$ is a subgroup of bounded exponent. Furthermore, for a given integer $n, D$ has only finitely many elements of order $n$.

Fact 8 (Zil'ber [Zi3]; see also [Po2 or Ta]). Let $G$ be a group of finite Morley rank. Let $H<G$ be an arbitrary subgroup of $G$. Denote by $\bar{H}$ the smallest definable subgroup of $G$ containing $H$. If $H$ is nilpotent (resp. solvable) then so is $\bar{H}$.

Recall that an element $e \in R$ of a ring $R$ is idempotent if $e \neq 0$ and $e^{2}=e$. An idempotent is atomic if for any other idempotent $f$, either $e f=0$ or $e f=e$.

Fact 9 (Cherlin-Reineke [Ch-R]). Let $R$ be a commutative ring of finite Morley rank with an identity. Then $R$ has finitely many atomic idempotents, say $e_{1}, \ldots, e_{n}$. These idempotents give rise to a canonical decomposition of $R$ (as a ring):

$$
R=R e_{1} \oplus \cdots \oplus R e_{n} .
$$

Each $R_{i}=R e_{i}$ is a local noetherian ring with definable nilpotent maximal ideal $M_{i} . M_{i}^{n} / M_{i}^{n+1}$ is a finite-dimensional vector space over $R_{i} / M_{i}$. If $R_{i}$ is infinite then $R_{i} / M_{i}$ is an algebraically closed field (by Fact 6).

\section{THE SOCLE}

For the rest of the article, unless otherwise stated, we assume that $G$ is a connected, solvable group of finite Morley rank in which $Z(G) \cap G^{\prime}=1$. In particular, $G$ is not nilpotent. Normal and definable subgroups of $G$ which are 
minimal for these properties will be called $G$-minimal subgroups. An infinite $G$-minimal subgroup is necessarily connected. If it is finite it must be central by Fact 1 . Thus finite ones intersect $G^{\prime}$ trivially. For infinite ones: either they are in $G^{\prime}$ or they intersect $G^{\prime}$ trivially. In the latter case they are central.

Let $S=S_{1}(G)$ be the subgroup generated by all the $G$-minimal subgroups of $G^{\prime}$. S will be called the socle of $G$. It is clearly a $G$-normal subgroup of $G^{\prime}$.

Proposition 1. $S=A_{1} \oplus \cdots \oplus A_{n}$ for some G-minimal subgroups $A_{i}$ of $G^{\prime}$. In particular, $S$ is definable and connected. Furthermore $S \subseteq Z\left(G^{\prime}\right)$.

Proof. By definition of the socle we have $S=\left\langle A_{i}: A_{i}\right.$ a $G$-minimal subgroup of $\left.G^{\prime}\right\rangle$. Since each $A_{i}$ is infinite (if not $A_{i} \subseteq Z(G) \cap G^{\prime}=1$ ) they are connected (if not $A_{i}^{\circ} \triangleleft G$ and $A_{i}^{\circ} \nsubseteq A_{i}$ ). Since $G^{\prime}$ is nilpotent (Fact 5) $Z\left(G^{\prime}\right) \cap A_{i} \neq 1$. $A_{i}$ being $G$-minimal, this forces $A_{i}$ to be in $Z\left(G^{\prime}\right)$. Thus $S \subseteq Z\left(G^{\prime}\right)$. Now we apply Fact 2, to get

$$
S=A_{1}+\cdots+A_{n}
$$

for some $G$-minimal $A_{i}$ 's. For $i=2, \ldots, n$, consider the subgroup

$$
A_{i} \cap\left(A_{1}+\cdots+A_{i-1}\right) .
$$

It is definable and normal. Since $A_{i}$ is $G$-minimal either $A_{i} \subset A_{1}+\cdots+A_{i-1}$, in which case we may delete $A_{i}$ from (1), or $A_{i} \cap\left(A_{1}+\cdots+A_{i-1}\right)=1$. Starting from $i=2$ and deleting unnecessary $A_{i}$ 's we get a direct sum.

We now fix our notation. For the rest of the article we will write

$$
S=A_{1} \oplus \cdots \oplus A_{n}
$$

where each $A_{i}$ is a $G$-minimal subgroup in $Z\left(G^{\prime}\right)$.

Lemma 2. Any nontrivial G-normal subgroup of $G^{\prime}$ intersects $S$ nontrivially.

Proof. Let $X$ be such a subgroup. Then the subgroup $[G, X]$ is connected by Fact 3. Since $X \triangleleft G,[G, X] \subseteq X$. Also $[G, X] \neq 1$ because if not $X \subseteq G^{\prime} \cap Z(G)=1$. Thus $[G, X]$ contains a $G$-minimal subgroup $A$ which must be in $S$. Thus $A \subseteq S \cap X$.

Let us show by an example that even if $G^{\prime}$ is abelian $S$ may be different from $G^{\prime}$.

Example 1. Let $K$ be an algebraically closed field. Let

$$
G=(K \times K) \rtimes\left(K[x] / x^{2}\right)^{*}
$$

where the action of $K[x] / x^{2}$ on $K \times K$ is given by

$$
(\alpha+\beta x)(a, b)=(\alpha a, \beta a+\alpha b),
$$


for $\alpha+\beta x \in K[x],(a, b) \in K \times K . \quad G$ is an algebraic group. A matrix representation of $G$ is

$$
G=\left\{\left(\begin{array}{cccc}
\alpha & \beta & 0 & b \\
0 & \alpha & 0 & a \\
0 & 0 & 1 & 0 \\
0 & 0 & 0 & 1
\end{array}\right): \alpha, \beta, a, b \in K, \alpha \neq 0\right\}
$$

$Z(G)=1, G^{\prime}=K \times K$, the socle of $G$ is $0 \times K$.

$\mathrm{Zil}^{\prime}$ ber proved in [Zi2] that each $A_{i}$ is isomorphic to the additive group $\left(F_{i},+\right)$ of an algebraically closed field $F_{i}$. He also obtained the multiplication of $F_{i}$ in $G$. We will now summarize the construction of the field $\left(F_{i},+, \cdot\right)$.

$A_{i}$ is a normal abelian subgroup of $G$. Thus $G$ acts on $A_{i}$ by conjugation. We can extend this action to the group ring $\mathbf{Z}[G]$ by

$$
\left(\sum_{i=1}^{k} n_{i} g_{i}\right)(a)=\prod_{i=1}^{k} a^{n_{i} g_{i}}
$$

for $\sum n_{i} g_{i} \in \mathbf{Z}[G], a \in A_{i}$. Since $A_{i}$ is abelian, the order of multiplication in formula (3) is not important. Now $A_{i}$ becomes a $\mathbf{Z}[G]$-module. Since $A_{i}$ is $G$-minimal, $A_{i}$ is an irreducible $\mathbf{Z}[G]$-module. Let us divide $\mathbf{Z}[G]$ by the annihilator of $A_{i}$. Thus $A_{i}$ is an irreducible, faithful $\mathbf{Z}[G] / \operatorname{Ann} A_{i}$-module. Set

$$
F_{i}=\mathbf{Z}[G] / \operatorname{Ann} A_{i} .
$$

$F_{i}$ is a commutative ring with 1 . By Schur's lemma $F_{i}$ has no 0 -divisors. In fact, if $\lambda \in F_{i}, \operatorname{Ker} \lambda=\left\{a \in A_{i}: \lambda a=0\right\}$ is a $G$-normal subgroup of $A_{i}$. Thus if $\lambda \neq 0, \operatorname{Ker} \lambda$ is finite thus 0 . Therefore each $\lambda \in F_{i} \backslash\{0\}$ is an automorphism of $A_{i}$ and is determined uniquely by $\lambda a$ for a fixed $a \in A_{i} \backslash\{0\}$. Zil'ber proved that $F_{i}$ is an interpretable field in $G$. More precisely, he proved that for some fixed integer $k_{i}$, any $\lambda \in F_{i}$ has a representative $\mu$ in $\mathbf{Z}[G]$ of length $\leq k_{i}$ (we remind the reader that the length of $\mu=\sum n_{i} g_{i} \in \mathbf{Z}[G], l(\mu)$, is $\left.\sum\left|n_{i}\right|\right)$. Thus by Fact $6, F_{i}$ is an algebraically closed field. In the sequel, the phrase "by Zil'ber" will refer to the above result.

Since for $a \in A_{i} \backslash\{0\}, F_{i} a$ is a definable $G$-normal subgroup of $A_{i}$, we have $F_{i} a=A_{i}$. Since Ann $_{F_{i}} a=0$ we have

$$
\left(F_{i},+\right) \simeq A_{i}
$$

Clearly $G / C_{G}\left(A_{i}\right)$ imbeds in $F_{i}^{*}$ and generates $F_{i}$ additively. The conjecture is that $G / C_{G}\left(A_{i}\right)=F_{i}^{*}$. This is saying that the fixed integer $k_{i}$ is 1 .

Now we give three examples to illustrate Zil'ber's method of finding the field. The examples will show that the construction may give rise to a field even if the group is unstable. 
Example 2. This is the easiest example to check Zil'ber's construction; let $K$ be any field and define

$$
G=K \rtimes K^{*}=\left\{\left(\begin{array}{ll}
1 & a \\
0 & b
\end{array}\right): a \in K, b \in K^{*}\right\} .
$$

Then

$$
A_{i}=S=G^{\prime}=\left\{\left(\begin{array}{ll}
1 & a \\
0 & 1
\end{array}\right): a \in K\right\} .
$$

Thus $n=1$. If $T$ is the set of diagonal matrices we have $T \simeq K^{*}$. In this example the integer $k$ is $1 . G$ is $\omega$-stable iff $K$ is algebraically closed, in which case the Morley rank of $G$ is 2 (assuming the language is $\left\{\cdot,{ }^{-1}\right.$, id $\}$ ).

Example 3. Let $H=\left\{\rho e^{2 \pi i \sigma}: \rho \in \mathbf{R}^{*}, \sigma \in \mathbf{Q}\right\} . H$ is a subgroup of $\left(\mathbf{C}^{*}, \cdot\right)$ Notice that $\mathbf{R}^{*}, i \mathbf{R}^{*} \subseteq H$. Thus any element of $\mathbf{C}$ can be written (not uniquely) as a sum of two elements of $H$. How consider the group

$$
G=\mathbf{C} \rtimes H=\left\{\left(\begin{array}{ll}
1 & a \\
0 & h
\end{array}\right): a \in \mathbf{C}, h \in H\right\} .
$$

Again $A_{i}=S=G^{\prime} \simeq \mathrm{C}, n=1$. But now the integer $k$ is 2 . However, $G$ can be shown not to have finite Morley rank. It is even unstable (one can define the field $\mathbf{R}$ ), as James Loveys and David Marker have recently shown.

Example 4. Let $S^{1}$ be the unit circle in $\mathrm{C}$, i.e.,

$$
S^{1}=\{\beta \in \mathbf{C}:|\beta|=1\} .
$$

Then $S^{1}<(\mathbf{C}, \cdot)$ and every element of $\mathbf{C}$ can be written as a sum of finitely many elements of $S^{1}$. But there is no bound in the number of elements of $S^{1}$ that we need to sum in order to get $\mathbf{C}$. Define

$$
G=\left\{\left(\begin{array}{ll}
1 & \alpha \\
0 & \beta
\end{array}\right): \alpha \in \mathbf{C}, \beta \in S^{1}\right\} \simeq \mathbf{C} \rtimes S^{1} .
$$

If we do Zil'ber's construction we find $A_{i}=S=G^{\prime}$, thus $n=1$, but now $k=\infty$. Thus $G$ does not have finite Morley rank. In this case one can prove that $G$ is unstable by interpreting the field $\mathbf{R}$ in $G$. Recently Marker generalized this result [Ma].

Above we explained $G$-actions on $G$-minimal subgroups. We may also look at the $G$-action on $S=A_{1} \oplus \cdots \oplus A_{n}$. We know that if we restrict thedaction to any of the $G$-minimal subgroups $A_{i}$ we find a field. But it may be that these restricted actions on $A_{i}$ are not independent. In [Ne2] we found all possible actions of $G$ on $S$. We will now explain this result.

We extend the action of $G$ on $S$ to $\mathrm{Z}[G]$ as in (3). Now $S$ becomes a $\mathrm{Z}[G] /$ Ann $S$-module. Let

$$
F=\mathbf{Z}[G] / \text { ann } S .
$$

$F$ also acts on each $A_{i}$. We clearly have natural isomorphisms

$$
F / \operatorname{ann}_{F} A_{i} \simeq F_{i} \text {. }
$$


But this is not enough to conclude that $F$ is interpretable in $G$. We proved in [Ne2] that $F$ is interpretable anyway. We need some notation before going further. Let

$$
M_{i}=\operatorname{ann}_{F} A_{i} .
$$

Since $F / M_{i} \simeq F_{i}$ and $F_{i}$ is a field, $M_{i}$ is a maximal ideal of $F$. Define an equivalence relation on the index set $\{1, \ldots, n\}$ by

$$
i \sim j \Leftrightarrow M_{i}=M_{j} \Leftrightarrow M_{i} \subseteq M_{j} .
$$

thus if $i \sim j$ then $F_{i}=F_{j}$. (The reader should be aware that this is an equality, more than an isomorphism.) Furthermore, in this case $F_{i}$ acts on $A_{i} \oplus A_{j}$ by scalar multiplication; i.e., for $\left(a_{i}, a_{j}\right),\left(a_{i}^{\prime}, a_{j}^{\prime}\right)$ in $A_{i} \oplus A_{j}$, for $\lambda \in F, \lambda\left(a_{i}, a_{j}\right)=\left(a_{i}^{\prime}, a_{j}^{\prime}\right)$ iff for some $\mu \in F_{i}=F_{j}$ we have $a_{i}^{\prime}=\mu a_{i}$, $a_{j}^{\prime}=\mu a_{j}$.

On the other hand, if $i \nsim j$ then $F$ acts on $A_{i} \oplus A_{j}$ "as freely as possible"; i.e., for $\left(a_{i}, a_{j}\right),\left(a_{i}^{\prime}, a_{j}^{\prime}\right) \in A_{i} \oplus A_{j}$, there is a $\lambda \in F$ for which $\lambda\left(a_{i}, a_{j}\right)=$ $\left(a_{i}^{\prime}, a_{j}^{\prime}\right)$ iff for some $\mu_{i} \in F_{i}, \mu_{j} \in F_{j}$

$$
a_{i}^{\prime}=\mu_{i} a_{i}, \quad a_{j}^{\prime}=\mu_{j} a_{j} .
$$

In other words,

$$
\begin{aligned}
& i \sim j \Leftrightarrow F / \operatorname{ann}\left(A_{i} \oplus A_{j}\right) \simeq F_{i}=F_{j}, \\
& i \not j \Leftrightarrow F / \operatorname{ann}\left(A_{i} \oplus A_{j}\right) \simeq F_{i} \times F_{j} .
\end{aligned}
$$

Let us give examples of both cases.

Example 5. Let $G=(K \times K) \rtimes K^{*}$ where $\alpha \in K^{*}$ acts on $K \times K$ by

$$
\alpha(a, b)=\left(\alpha a, \alpha^{2} b\right) .
$$

Then $G$ is an algebraic group. It is connected, solvable, and centerless. $G^{\prime}=$ $K \times K, S=G^{\prime}, n=2, A_{1}=K \times\{0\}, A_{2}=\{0\} \times K, F_{1} \simeq K \simeq F_{2}$. Let us find $F$. Let us first suppose that char $K \neq 2$. Let $i \in K$ be an element for which $i^{2}=-1$. Imbed $K^{*}$ in $G$ and $\mathbf{Z}\left[K^{*}\right]$ in $\mathbf{Z}[G]$. Consider the element $(i)+(1)$ of $\mathbf{Z}[G]$ (which is different from the element $(1+i) \in K$ of $\mathbf{Z}[G]$ ). If $(a, b) \in K \times K$ then

$$
((i)+(1))(a, b)=i(a, b)+1(a, b)=(i a,-b)+(a, b)=((1+i) a, 0) .
$$

Thus $(i)+(1)$ acts as the 0 endomorphism on $A_{2}=\{0\} \times K$ but not on $A_{1}$.

Next consider the element $(1)+(-1)$ of $\mathbf{Z}[G]$. If $(a, b) \in K \times K$ then

$$
((1)+(-1))(a, b)=1(a, b)+(-1)(a, b)=(a, b)+(-a, b)=(0,2 b) \text {. }
$$

Thus $(1)+(-1)$ acts as the 0 endomorphism on $A_{1}$ but not on $A_{2}$. Therefore in this case we have two equivalence classes. By using these two endomorphisms it is easy to show that $F \simeq K \times K$ and it acts on $S=K \times K$ componentwise. 
If Char $K=2$, then $G$ is isomorphic to the group described in the next example.

Example 6. Let $G=(K \times K) \rtimes K^{*}$ with the following action:

$$
\alpha(a, b)=(\alpha a, \alpha b)
$$

for $\alpha \in K^{*},(a, b) \in K \times K$. We have $S=G^{\prime}=K \times K, n=2, A_{1}=\{0\} \times K$, $A_{2}=K \times\{0\} . \quad F_{1} \simeq K \simeq F_{2}$. But in this case $F \simeq K$ also (because we have only one equivalence class).

In [Ne2] we showed that these two examples are essentially the only possible actions of $\mathbf{Z}[G]$ on $S$. Let us make this more precise.

We first reindex

$$
S=\left(A_{11} \oplus \cdots \oplus A_{1 m_{1}}\right) \oplus \cdots \oplus\left(A_{l 1} \oplus \cdots \oplus A_{l m_{l}}\right)
$$

in such a way that if

$$
B_{i}=A_{i 1} \oplus \cdots \oplus A_{i m_{i}}
$$

then

$$
A_{i} \subseteq B_{i}
$$

and

$$
A_{j}, A_{k} \subseteq B_{i} \Leftrightarrow j \sim k .
$$

In other words, we assemble in a block all the $A_{i}$ 's which have the same annihilators in $\mathbf{Z}[G]$. Thus (with the conventions (11) and (12)), $\operatorname{Ann}\left(A_{i}\right)=$ $\operatorname{Ann}\left(B_{i}\right)=M_{i}$. Now $F_{i}$ acts on $B_{i}$ and $B_{i}$ becomes a vector space over $F_{i}$.

Fact (see [Ne2], claims 8, 9, and 10).

(A) Let $a=\left(a_{i 1}, \ldots, a_{i m_{i}}\right), a^{\prime}=\left(a_{i 1}^{\prime}, \ldots, a_{i m_{i}}^{\prime}\right)$ be two elements of $B_{i}$. Then there is a $\beta \in F$ for which $\beta a=a^{\prime}$ iff there is a $\gamma \in F_{i}$ for which $\gamma a_{i j}=a_{i j}^{\prime}$ for all $j=1, \ldots, m_{i}$.

(B) Let $b=\left(b_{1}, \ldots, b_{l}\right), b^{\prime}=\left(b_{1}, \ldots, b_{l}^{\prime}\right) \in S$ with $b_{i}, b_{i}^{\prime} \in B_{i}$. Then there is a $\beta \in F$ for which $\beta b=b^{\prime}$ iff there are $\gamma_{i} \in F_{i}(i=1, \ldots, l)$ for which $\gamma_{i} b_{i}=b_{i}^{\prime}$ for all $i=1, \ldots, l$.

(C) There is an integer $k$ such that any $\beta \in F$ has a representative $\gamma \in \mathbf{Z}[G]$ of length $\leq k$. In other words, the ring $F$ is interpretable in $G$.

Facts A and B say that $F \simeq F_{1} \times \cdots \times F_{l}$. We can state these two facts in one compact form:

(D) $S \rtimes F^{*}=\bigoplus_{i=1}^{l}\left(B_{i} \rtimes F_{i}^{*}\right)$ and

$$
B_{i} \rtimes F_{i}^{*} \simeq\left\{\left(\begin{array}{ll} 
& a_{i_{1}} \\
\operatorname{Id} & \vdots \\
& a_{i m_{i}}
\end{array}\right) a_{i j} \in A_{i j}, \beta \in F_{i}^{*}\right\} .
$$


In particular, each group $B_{i} \rtimes F_{i}^{*}$ is an algebraic group over an algebraically closed field.

Next, we state some immediate consequences of Facts A, B, C, and D.

Lemma 3. Every G-normal subgroup of $S$ is definable and connected.

Proof. Since $F=F_{1} \times \cdots \times F_{l}$ and each $F_{i}$ is connected (Fact 6), $F$ is a connected ring.

Now let $\mathbf{U}$ be a $G$-normal subgroup of $S$. Let $x \in \mathbf{U}$. Clearly $F x \simeq$ $F / \operatorname{ann}_{F}(x)$. Thus $F x$ is a definable connected subgroup. Since $F$ has an identity element, $x \in F x$. Thus

$$
\mathbf{U}=\langle F x: x \in \mathbf{U}\rangle \text {. }
$$

By Fact 2, $\mathbf{U}$ is definable and connected.

Lemma 4. $S$ is a completely reducible F-module.

Proof. Let $\mathbf{U}$ be an $F$-submodule of $S$. In particular, $\mathbf{U}$ is $G$-normal. Thus by Lemma 3, it is definable. We will find a complement $V$ to $\mathbf{U}$ in $S$. If for all $G$-minimal $A, A \subseteq \mathbf{U}$, then there is nothing to do, because then $\mathbf{U}=S$, $V=\{0\}$. If not, for some $G$-minimal $A, A \cap \mathbf{U}=\{0\}$. Thus $\langle\mathbf{U}, A\rangle=\mathbf{U} \oplus A$. Since $\operatorname{rk}(S / \mathbf{U} \oplus A)$ is strictly less than $\operatorname{rk}(S / \mathbf{U})$, by induction, $\mathbf{U} \oplus A$ has a complement in $S$.

In $\S 6$ we will generalize Lemma 3 to $G^{\prime}$ in case $G^{\prime}$ is abelian (see Corollary 19).

\section{SPlitting $G^{\prime}$}

The purpose of this section is to prove Theorems 1 and 2 stated in the Introduction. We keep the same hypothesis about $G$ : it is connected, solvable, has finite Morley rank, and $G^{\prime} \cap Z(G)=1$. We will use the notation of $\S 4$.

Lemma 5. Suppose $G$ is $\omega_{1}$-saturated. Then there is an element $g \in G$ whose order modulo each $C_{G}\left(A_{i}\right)$ is infinite.

Proof. If the lemma does not hold then for all $g \in G$ there is an integer $p=p(g)$ such that $g^{p} \in \bigcup_{i=1}^{n} C_{G}\left(A_{i}\right)$. Since $G$ is saturated the same integer $p$ will work for all $g \in G$. Thus $g^{p} \in \bigcup_{i=1}^{n} C_{G}\left(A_{i}\right)$ for all $g \in G$. Let

$$
G_{i}=\left\{g: g^{p} \in C_{G}\left(A_{i}\right)\right\} \text {. }
$$

Thus

$$
G=G_{1} \cup \cdots \cup G_{n} .
$$

Therefore $\operatorname{rk} G=\operatorname{rk} G_{i}$ for some $i$. But $G_{i}$ is a subgroup (because $G^{\prime} \subseteq$ $C_{G}\left(A_{i}\right) \subseteq G_{i}$ and $G / C_{G}\left(A_{i}\right)$ is abelian). Since $G$ is connected $G=G_{i}$. But then $G / C_{G}\left(A_{i}\right)$ is a group of exponent $p$. Also $G / C_{G}\left(A_{i}\right)<F_{i}^{*}$. Since $F_{i}$ is a field it has finitely many elements of order $p$. Hence $G / C_{G}\left(A_{i}\right)$ is finite. $G$ being connected, this implies $G=C_{G}\left(A_{i}\right)$, i.e., $A_{i} \subseteq G^{\prime} \cap Z(G)=1$, a contradiction. This proves the lemma. 
Remark. Since $C_{G}\left(A_{i}\right) \neq G$ and $G$ is connected, $G \neq \bigcup_{i=1}^{n} C_{G}\left(A_{i}\right)$. So it is easy to find a $g$ not in any of the centralizers. Lemma 6 says that, if $G$ is $\omega_{1}$-saturated, we can find a $g \in G \backslash \bigcup_{i=1}^{n} C_{G}\left(A_{i}\right)$ with the property that $g^{p} \notin$ $C_{G}\left(A_{i}\right)$ for any $i=1, \ldots, n$, any $p \geq 1$.

Lemma 6. Let $g \in G \backslash \bigcup_{i=1}^{n} C_{G}\left(A_{i}\right)$. Then $C_{S}(g)=1$.

Proof. Since $S=\bigoplus_{i=1}^{n} A_{i}$ and $A_{i} \triangleleft G$, we have $C_{S}(g)=\bigoplus_{i=1}^{n} C_{A_{i}}(g)$. Thus we just need to show that $C_{A_{i}}(g)=1$. We will show that $C_{A_{i}}(g) \triangleleft G$. Since $A_{i}$ is $G$-minimal this will imply that either $C_{A_{i}}(g)=A_{i}$ or $C_{A_{i}}(g)=1$. But $C_{A_{i}}(g)=A_{i}$ means $g \in C_{G}\left(A_{i}\right)$, which contradicts the choice of $g$. So let us show $C_{A_{i}}(g) \triangleleft G$. Let $a \in C_{A_{i}}(g), h \in G$. Since by Proposition $1 G^{\prime} \subseteq$ $C_{G}\left(A_{i}\right)$ we have

$$
a^{h}=a^{g h}=a^{h g} .
$$

Thus $a^{h} \in C_{A_{i}}(g)$ and we are done.

From now on we fix such a $g$.

We would like to be able to do induction on the rank of $G$. But if $\bar{G}=G / S$ we may have $Z(\bar{G}) \cap \bar{G}^{\prime} \neq 1$. This will keep us away from the hypothesis. Let us consider $Z(\bar{G})$ and see what conditions we need on $G$ for $Z(\bar{G}) \cap \bar{G}^{\prime}$ to be the trivial subgroup of $\bar{G}$. Let

$$
X=\left\{x \in G^{\prime} \mid[G, x] \subseteq S\right\} .
$$

Then $X$ is a $G$-normal definable subgroup of $G^{\prime}$ containing $S$. In fact,

$$
X / S=(G / S)^{\prime} \cap Z(G / S) .
$$

(Recall that $(G / S)^{\prime}=G^{\prime} S / S=G^{\prime} / S$.)

Lemma 7. $X=S \oplus C_{X}(g)$.

Proof. Since $S \subseteq Z\left(G^{\prime}\right)$ and $C_{X}(g) \subseteq G^{\prime}$ the additive notation $\oplus$ makes sense. We will switch from multiplicative to additive notation without any comment.

Clearly, $S+C_{X}(g) \subseteq X$. By Lemma $6, S+C_{X}(g)=S \oplus C_{X}(g)$.

Conversely, let $x \in X$. Then by definition of $X,[g, x] \in S$. On the other hand, by the previous lemma $C_{S}(g)=1$; thus the group homomorphism

$$
\tilde{g}: S \rightarrow S
$$

defined by

$$
\tilde{g}(s)=[g, s]
$$

is an automorphism. Thus

$$
[g, x]=[g, s]
$$

for some $s \in S$, i.e.,

$$
s^{-1} x \in C_{X}(g)
$$

for some $s \in S$. Hence $x=s s^{-1} x \in S \oplus C_{X}(g)$. This proves the lemma. 
Lemma 8. If $C_{X}(g) \triangleleft G^{\prime}$ then $C_{X}(g)=1$.

Proof. We will prove that $C_{X}(g) \triangleleft G^{\prime}$ implies $C_{X}(g) \triangleleft G$. This will prove the lemma in view of Lemmas 2 and 6. Let $c \in C_{X}(g), h \in G$. Since $[h, g] \in G^{\prime}$, by the definition of $X$,

$$
[c,[h, g]] \in C_{X}(g) \cap S=1 .
$$

Thus

$$
c^{h g}=c^{g h}=c^{h} \text {. }
$$

This shows that $c^{h} \in C_{X}(g)$. Since this is true for all $c \in C_{X}(g), h \in G$, we have $C_{X}(g) \triangleleft G$.

Corollary 9. If $G$ is solvable of class 2 then $Z(G / S) \cap\left(G / S^{\prime}\right)=1$.

Proof. By hypothesis $G^{\prime}$ is abelian. So $C_{X}(g) \triangleleft G^{\prime}$. By Lemma $8, C_{X}(g)=1$. By Lemma 7, $X=S$. Since $1=X / S=Z(G / S) \cap G^{\prime} / S$, we have the lemma.

Lemma 10. If for some $t \in G, C_{G^{\prime}}(t)=1$, then $C_{\bar{G}^{\prime}}(t)=1$ where $\bar{G}=G / S$. Proof. Let $Y=\left\{y \in G^{\prime}:[y, t] \in S\right\} . Y$ is a definable subgroup of $G$. In fact

$$
Y / S=C_{\bar{G}^{\prime}}(t) .
$$

Let $y \in Y$. So $[y, t] \in S$. On the other hand,

$$
C_{S}(t) \subseteq C_{G^{\prime}}(t)=1 ;
$$

thus the homomorphism $\tilde{t}$ defined by

$$
\tilde{t}: S \rightarrow S, \quad \tilde{t}(s)=[s, t]
$$

is in fact an automorphism. Therefore for some $s \in T$

$$
[y, t]=[s, t],
$$

i.e., $s^{-1} y \in C_{G^{\prime}}(t)=1$, so $s=y$. This shows that $Y=S$, so $C_{\bar{G}^{\prime}}(t)=1$.

We next show that in order to split $G^{\prime}$ in $G$, it is enough to split $(G / S)^{\prime}$ in $G / S$. We say that $G^{\prime}$ splits definably in $G$ if there is a definable subgroup $T$ for which $G=G^{\prime} \rtimes T$.

Proposition 11. If $(G / S)^{\prime}$ splits definably in $G / S$ then $G^{\prime}$ splits definably in $G$.

Proof. Suppose $G / S=(G / S)^{\prime} \rtimes(T / S)$ for some definable subgroup $T$ containing $S$. Since $(G / S)^{\prime}=G^{\prime} / S$ we have

(i) $G=G^{\prime} T$,

(ii) $G^{\prime} \cap T=S$.

We will split $S$ definably in $T$. More specifically, we will show that

$$
T=S \rtimes C_{T}(t)
$$


for some $t$ in $T$. Let $t$ be an element of $T$ for which $C_{S}(t)=1$ (we know there is such an element in $G$; by (i) we may take it in $T$ ). As in the proof of Lemma 10 we have

$$
S=\{[s, t]: s \in S\} .
$$

Let $t_{1} \in T$ be any element. Then

$$
\left[t_{1}, t\right] \in T^{\prime} \subseteq G^{\prime} \cap T=S .
$$

Thus $\left[t_{1}, t\right] \in S$. So for some $s \in S$

$$
\left[t_{1}, t\right]=[s, t],
$$

i.e., for some $s \in S, s^{-1} t, \in C_{T}(t)$. Now we can write

$$
t_{1}=s s^{-1} t_{1}
$$

with $s \in S, s^{-1} t_{1} \in C_{T}(t)$. So

$$
T=S C_{T}(t)
$$

Clearly

$$
S \cap C_{T}(t)=C_{S}(t)=1 .
$$

This proves the proposition, becuase now $G=G^{\prime} \rtimes C_{T}(t)$.

We can now prove the first two theorems stated in the introduction.

Theorem 1. Let $G$ be a connected, solvable group of class $\leq 2$ and of finite Morley rank in which $Z(G) \cap G^{\prime}=1$. Then for some definable subgroup $T$, $G=G^{\prime} \rtimes T$.

Proof. The theorem easily follows from Corollary 9 and Proposition 11 by induction on the rank of $G$. We first split $G^{\prime} / S$ in $G / S$ (using Corollary 9) and apply Proposition 11 .

Theorem 2. Let $G$ be a connected, solvable group of finite Morley rank. Assume for some $t \in G, C_{G^{\prime}}(t)=1$. Then there is a definable subgroup $T$ of $G$ such that

$$
G=G^{\prime} \rtimes T .
$$

Proof. Using the inductive method of proof on the rank of $G$, the theorem follows from Lemma 10 and Proposition 11 . We first split $G^{\prime} / S$ in $G / S$ (using Lemma 10) and apply Proposition 11.

Remark 1. $T$ of the above theorems is necessarily connected.

Remark 2. Since $T^{\prime} \subseteq T \cap G^{\prime}=1, T$ is Abelain.

Remark 3. If $G$ is an algebraic group, the subgroup $T$ of the theorems may be bigger than a maximal torus; i.e., it may contain unipotent elements. In Example 1, e.g.,

$$
T=\left(K[x] / x^{2}\right)^{*}
$$


and the elements of the form

$$
1+\alpha x
$$

of $T$ are unipotent elements. The algebraic torus in this case is the set

$$
\left\{\beta+0 \cdot x: \beta \in K^{*}\right\} \simeq K^{*} \text {. }
$$

We suspect that if Char $K=0$ the torus is not definable. However, it is interpretable as a quotient of $G$ by the maximal nilpotent subgroup (see $\S 8$ for the definability of this maximal nilpotent subgroup).

Corollary 12. Suppose $G$ is as in Theorem 1. Assume furthermore that $Z(G)=$ 1 and $G^{\prime}=S$. Then $G$ can be interpretably imbedded in a finite product $\widetilde{G}$ of interpretable algebraic groups. $\widetilde{G}$ has the form of the group $S \rtimes F^{*}$ described in Fact D.

Proof. Let $G=G^{\prime} \rtimes T$. Since $T$ is an abelian subgroup, $C_{T}\left(G^{\prime}\right)$ commutes with $T$. It obviously commutes with $G^{\prime}$. Thus

$$
C_{T}\left(G^{\prime}\right) \subseteq Z(G)=1 \text {. }
$$

Thus $T<F^{*}$ (notation as in $\S 4$ ). In fact, $\mathrm{Z}[T] /$ ann $S$ is naturally isomorphic to $F$. Since $G=G^{\prime} \rtimes T \subseteq G^{\prime} \rtimes F^{*}$, we obtain the corollary from Facts $\mathrm{C}$ and D.

Remark. Even if $G$ is an algebraic group satisfying the hypothesis of Corollary 1, $\widetilde{G}$ may be strictly bigger than $G$. In Example 5, e.g., $\widetilde{G}=(K \times K) \rtimes\left(K^{*} \times K^{*}\right)$ with the componentwise action.

Conjecture. Any centerless, connected, solvable group of finite Morley rank can be intepretably imbedded in a finite product of interpretable, (centerless, connected, solvable), algebraic groups over algebraically closed fields.

See $[\mathrm{E}-\mathrm{N}]$ for a partial solution of the Conjecture.

Corollary 13. Suppose $G$ is as in Theorem 1. Then $C_{G}\left(G^{\prime}\right)=G^{\prime}$.

Proof. We have $G=G^{\prime} \rtimes T$. Since $G^{\prime}$ is abelian, $G^{\prime} \subseteq C_{G}\left(G^{\prime}\right)$. Thus $C_{G}\left(G^{\prime}\right)=G^{\prime} \rtimes\left(T \cap C_{G}\left(G^{\prime}\right)\right)$. But $T \cap C_{G}\left(G^{\prime}\right)$ commutes with $T$ (because $T$ is abelian). It also commutes with $G^{\prime}$; thus $C_{G}\left(G^{\prime}\right) \cap T \subseteq Z(G)=1$. Hence $C_{G}\left(G^{\prime}\right)=G^{\prime}$.

Let us show by an example that the hypotheses of Theorems 1 and 2 are crucial. We would like to thank Xialong $\mathrm{Wu}$ for providing the example.

Example 7. Let $G$ be the subgroup of invertible upper-triangular $6 \times 6$ matrices over a field $K$ with $a_{11}=a_{22}=a_{33}$ and $a_{44}=a_{55}=a_{66}$, i.e.,

$$
G=\left\{\left(\begin{array}{cccccc}
a & & & & \\
& a & & * & \\
& & a & & & \\
& & & b & & \\
& & & b & \\
& 0 & & & b
\end{array}\right) \mid a, b \in K^{*}\right\} .
$$


Then $G^{\prime}$ is the set of strictly-triangular matrices with $a_{12}=a_{23}=a_{45}=$ $a_{56}=0$, i.e.,

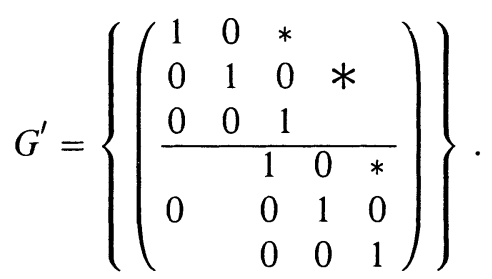

$G^{\prime}$ has no complement in $G$ (definable or not). This is an example where $G^{\prime}$ is not abelian, $C_{G^{\prime}}(g) \neq 1$ for all $g$, and $Z(G) \cap G^{\prime}=1$. To find a centerless counterexample divide $G$ by its center.

Definition. Let $G$ be a connected solvable group of finite Morley rank in which $Z(G) \cap G^{\prime}=1$. Assume furthermore that either

(1) $G^{\prime}$ is abelian or

(2) for some $g \in G, C_{G^{\prime}}(g)=1$.

In Lemmas 9 and 10 , we showed that $G / S$ satisfies the same properties; in particular $Z(G) \cap G^{\prime}=1$ where $G=G / S$. Thus we can define $S_{2}=S_{2}(G)$ by

$$
S_{2} / S_{1}=S\left(G / S_{1}\right)
$$

where $S_{1}=S$. In general we set

$$
S_{i+1} / S_{i}=S\left(G / S_{i}\right)
$$

and we call $S_{i}$ the $i$ th socle of $G$. Since each $S_{i}$ is connected and we have $S_{i} \subseteq$ $S_{i+1}$, for some integer $j, S_{j}=G^{\prime}$. By Corollary 12, if $T$ is a complement of $G^{\prime}$ in $G$, the group $\left(S_{i+1} / S_{i}\right) \rtimes T$ modulo a subgroup of $T$ imbeds interpretably in a product of interpretable algebraic groups (described in Fact D).

\section{SOlVABLE GROUPS OF ClASS 2}

We assume in this section that $G$ is a connected, centerless 2-step solvable group of finite Morley rank. Notation is as in the previous sections. $S$ denotes the socle of $G$. We have $S=A_{1} \oplus \cdots \oplus A_{n}$ for $G$-minimal subgroups $A_{i} . T$ is a complement of $G^{\prime}$ in $G: G=G^{\prime} \rtimes T . \mathrm{Z}[T] /$ ann $A_{i}=F_{i}$ is an algebraically closed field. $\mathrm{Z}[T] /$ ann $S=F$ is a ring which is isomorphic to a direct sum of some of the $F_{i}$ 's. The group $S \rtimes F^{*}$ was described in Facts A, B, C, and D.

We now consider the action of $T$ on $G^{\prime}$. Since $G^{\prime}$ is an Abelian group we may make $\mathrm{Z}[T]$ act on $G^{\prime}$. Let $R=\mathrm{Z}[T] /$ ann $G^{\prime}$. Clearly $R$ also acts on $S$ and $A_{i}$. It should be clear that we have the following canonical isomorphisms:

$$
R / \operatorname{ann}_{R} S \simeq F, \quad R / \operatorname{ann}_{R} A_{i} \simeq F_{i} .
$$

By Corollary 13, $T<R^{*}$. Therefore our group $G=G^{\prime} \rtimes T$ imbeds naturally in $\widetilde{G}=G^{\prime} \rtimes R^{*}$. We call $\widetilde{G}$ the full group of $G$. We will show that the group $\widetilde{G}$ is interpretable in $G$. Then $\widetilde{G}$ will also have finite Morley rank.

Let us find the full group of some of the examples we gave. 
In Examples 1, 2, and 6, $\widetilde{G}=G$.

In Example 3, $\widetilde{G}=\mathbf{C} \times \mathbf{C}^{*}=\left\{\left(\begin{array}{ll}1 & a \\ 0 & b\end{array}\right): a, b \in \mathbf{C}, b \neq 0\right\}$.

In Example 5, $\widetilde{G}=(\mathbf{C} \times \mathbf{C}) \rtimes\left(\mathbf{C}^{*} \times \mathbf{C}^{*}\right)$.

Already Example 3 shows that $\widetilde{G}$ may look more like an algebraic group than $G$. Clearly $\widetilde{\widetilde{G}}=\widetilde{G}$ and $\widetilde{G}^{\prime}=G^{\prime}$.

The section is devoted to the proof of the fact that $R$ is interpretable in $G$. By trying to prove this we obtain a lot of information about the action of $R$ on $G^{\prime}$.

Lemma 14. Let $x \in S$. Then

(i) $R x=F x$,

(ii) $R x$ is a connected normal subgroup of $G$,

(iii) $R x$ is a direct sum of $G$-minimal subgroups.

Proof. Since $x \in S$ and $R / \operatorname{ann}_{R} S \simeq F$ (naturally), (i) follows. (ii) and (iii) are immediate from Lemmas 3 and 4.

Since $S_{i}$ is $G$-normal, $S_{i}$ is also an $R$-module.

Lemma 15. Let $x \in S_{i+1}$. Then

(i) $\left(S_{i}+R x\right) / S_{i}$ is a connected, $G / S_{i}$-normal subgroup of $G^{\prime} / S_{i}$,

(ii) $\left(S_{i}+R x\right) / S_{i}$ is a direct sum of $G / S_{i}$-minimal subgroup of $G^{\prime} / S_{i}$.

Proof. We may proceed as in Lemma 14 (replacing $G$ by $G / S_{i}$ ), if we can show that $G / S_{i}$ satisfies the hypothesis of Lemmas 3 and 4 . This is done in Corollary 9.

Lemma 16. Let $x \in G^{\prime}$. Then $R x$ is a connected, G-normal subgroup of $G^{\prime}$. Proof. We will prove by induction on $i$ that $R x \cap S_{i}$ is connected. Since each $x \in G^{\prime}$ is in some $S_{i}$ this will prove the first claim of the lemma. The second claim is obvious.

Since $1 \in R$ we have

$$
\left(S_{i-1}+\left(R x \cap S_{i}\right)\right) / S_{i-1}=\left\langle R \bar{y} \mid y \in R x \cap S_{i}\right\rangle
$$

where $\bar{y}$ is the image of $y$ in $G / S_{i-1}$. By Lemma 15 , each $R \bar{y}$ is connected. By Fact 2, the group generated by these $R \bar{y}$ 's is connected. Thus

$$
\left(S_{i-1}+\left(R x \cap S_{i}\right)\right) / S_{i-1} \simeq\left(R x \cap S_{i}\right) /\left(R x \cap S_{i-1}\right)
$$

is connected. But by induction $R x \cap S_{i-1}$ is connected. These easily imply that $R x \cap S_{i}$ is connected.

The next lemma gives explicitly the definition of $R x$ (or equivalently of $\left.R / \operatorname{ann}_{R} x\right)$.

Lemma 17. Let $x \in G^{\prime}$. There is an integer $k$ for which

$$
R x=\{r(x): r \in \mathbf{Z}[T] \text { and } l(r) \leq k\} .
$$

Hence $R /$ ann $x$ is an intrepretable ring. 
Proof. Suppose $x \in S_{i} \backslash S_{i-1}$. Let $\bar{x} \in G / S_{i-1}$ be the image of $x$. By Fact C, for some integer $k_{0}$,

$$
R \bar{x}=\left\{r(\bar{x}): r \in \mathbf{Z}[T] \text { and } l(r) \leq k_{0}\right\} .
$$

Thus for all $r \in \mathbf{Z}[T]$, there is an $r^{\prime} \in \mathbf{Z}[T]$ of length $\leq k_{0}$ such that

$$
r(x)-r^{\prime}(x) \in R x \cap S_{i-1} .
$$

On the other hand, by Fact 2 and Lemma 16,

$$
R x \cap S_{i-1}=\left\langle R y: y \in R x \cap S_{i-1}\right\rangle=R y_{1}+\cdots+R y_{u}
$$

for some $y_{1}, \ldots, y_{u} \in R x \cap S_{i-1}$. Write $y_{j}=r_{j}(x)$, so

$$
R x \cap S_{i-1}=R r_{1}(x)+\cdots+R r_{u}(x) .
$$

Thus $r(x)-r^{\prime}(x) \in R r_{1}(x)+\cdots+R r_{u}(x)$. But $r_{j}(x) \in S_{i-1}$, so by induction (on $i$ ) for some integer $k_{j}$,

$$
\begin{aligned}
R r_{j}(x) & =\left\{r r_{j}(x): l(r) \leq k_{j}, r \in \mathbf{Z}[T]\right\} \\
& =\left\{s(x): l(s) \leq k_{j}+l\left(r_{j}\right), s \in \mathbf{Z}[T]\right\} .
\end{aligned}
$$

Hence $r(x)=r^{\prime}(x)+s_{1}(x)+\cdots+s_{n}(x)$ where $r^{\prime}, s_{j} \in \mathbf{Z}[T], l\left(r^{\prime}\right) \leq k_{0}$, $l\left(s_{j}\right) \leq k_{j}+l\left(r_{j}\right)$. If $r^{\prime \prime}=r^{\prime}+s_{1}+\cdots+s_{u}$ we have $r(x)=r^{\prime \prime}(x)$ and $l\left(r^{\prime \prime}\right) \leq k_{0}+$ $\cdots+k_{n}+l\left(r_{1}\right)+\cdots+l\left(r_{n}\right)$. Set $k$ to be the integer $k_{0}+\cdots+k_{n}+l\left(r_{1}\right)+\cdots+l\left(r_{n}\right)$.

Since an element $r \in R / \operatorname{ann} x$ is uniquely defined by $r(x)$, the last claim is now obvious.

The above lemma does not say that $R$ is interpretable in $G$. It just states that for any $x \in G^{\prime}, R / \operatorname{ann}_{R} x$ is interpretable in $G$. We will prove that $R$ is indeed interpretable in $G$. Let us first give an example where $G^{\prime} \neq$ $R x_{1} \oplus \cdots \oplus R x_{u}$ for any $x_{1}, \ldots, x_{u} \in G^{\prime}$.

Example 8. Let $G=(K \times K \times K) \rtimes\left(K[x] / x^{2}\right)^{*}$ with the following action:

$$
(\alpha+\beta x)(a, b, c)=(\alpha a, \alpha b, \alpha c+\beta a+\beta b) .
$$

Then

$$
\begin{aligned}
& S=0 \times 0 \times K, \\
& G^{\prime}=K \times K \times K, \\
& R=K[x] / x^{2}, \\
& R(1,0,0)=K \times 0 \times K, \\
& R(0,1,0)=0 \times K \times K, \\
& G^{\prime}=R(1,0,0)+R(0,1,0) .
\end{aligned}
$$

Since $\operatorname{dim} G^{\prime}=3, \operatorname{dim} R=2$, there is no $x \in G^{\prime}$ for which $R x=G^{\prime}$. For the same reason there are no $x_{1}, x_{2} \in G^{\prime}$ for which $G^{\prime}=R x_{1} \oplus R x_{2}$. 
Lemma 18. $G^{\prime}=R x_{1}+\cdots+R x_{u}$ for some $x_{1}, \ldots, x_{u} \in G^{\prime}$.

Proof. Since $1 \in R, G^{\prime}=\left\langle R x \mid x \in G^{\prime}\right\rangle$. By Fact 2 (using Lemma 16),

$$
G^{\prime}=R x_{1}+\cdots+R x_{u}
$$

for some $x_{1}, \ldots, x_{u} \in G^{\prime}$.

The next result is an unexpected property of $G$ :

Corollary 19. Any G-normal subgroup of $G^{\prime}$ is definable and connected. Proof. Let $X \subseteq G^{\prime}$ be a $G$-normal subgroup. Since $1 \in R$, we have

$$
X=\langle R x \mid x \in X\rangle .
$$

By Lemma 16, each $R x$ is connected. So by Fact $2, X$ is definable and connected.

We say that $x \in G^{\prime}$ is a minimal element (of $S_{i}$ ) if $x \in S_{i} \backslash S_{i-1}$ and if $\left(R x+S_{i-1}\right) / S_{i-1}$ is a $G / S_{i-1}$-minimal subgroup. Notice that if $x$ is a minimal element of $S_{i}$ then $\left(R x+S_{i-1}\right) / S_{i-1}$ is isomorphic to the additive structure of the algebraically closed field $R / M_{x}$ where

$$
M_{x}=\left\{r \in R: r(x) \in S_{i-1}\right\} .
$$

This is the content of Zil'ber's construction.

We say that $x \in G^{\prime}$ is a local element if $x$ is minimal and if $R / \operatorname{ann} x$ is a local ring.

By Fact 9 and Lemma 17 if $x$ is a local element then the maximal ideal of $R /$ ann $x$ is definable and nilpotent. Thus $R / \operatorname{ann} x$ cannot have a nontrivial idempotent.

Notice that the local elements of $S_{1}$ are exactly the minimal elements of $S_{1}$.

Lemma 20. If $x \in S_{i} \backslash S_{i-1}$ is minimal then there is a local element $y \in R x$ such that

$$
\left(R x+S_{i-1}\right) / S_{i-1}=\left(R y+S_{i-1}\right) / S_{i-1} .
$$

Proof. Let $\bar{R}=R /$ ann $x$. We know by Lemma 17 that $\bar{R}$ is interpretable in $G$. Thus it has finite Morley rank. Hence by Fact $9, \bar{R}=\bar{R} \bar{e}_{1} \oplus \cdots \oplus \bar{R} \bar{e}_{t}$ for orthogonal atomic idempotents $\bar{e}_{i} \in \bar{R}$, each $\bar{R} \bar{e}_{j}$ being a local ring. Now we can decompose $R x$ accordingly:

$$
R x=R e_{1} x \oplus \cdots \oplus \operatorname{Re}_{t} x .
$$

Since $\left(R x+S_{i-1}\right) / S_{i-1}$ is $G / S_{i-1}$-minimal, all but one of the elements $e_{j} x$ are in $S_{i-1}$, say $e_{1} x \notin S_{i-1}$. Then

$$
\left(R x+S_{i-1}\right) / S_{i-1}=\left(\operatorname{Re}_{1} x+S_{i-1}\right) / S_{i-1} .
$$

It remains to show that $R / \operatorname{ann}\left(e_{1} x\right)$ is a local ring. This is the same as showing $\bar{R} / \operatorname{ann}\left(\bar{e}_{1} \bar{x}\right)$ is a local ring. But clearly $\bar{R} / \operatorname{ann}\left(\bar{e}_{1} \bar{x}\right) \simeq \bar{R} \bar{e}_{1}$. 
Lemma 21. Let $x \in G^{\prime}$ be a local element. Then the maximal ideal of $R /$ ann $x$ is $M_{x} /$ ann $x$. Also $M_{x} /$ ann $x$ is an interpretable nilpotent ideal.

Proof. $M_{x} /$ ann $x$ is certainly a proper ideal of $R /$ ann $x$. Since

$$
(R / \operatorname{ann} x) /\left(M_{x} / \operatorname{ann} x\right) \simeq R / M_{x}
$$

and the latter is a field, $M_{x} /$ ann $x$ is the maximal ideal of $R /$ ann $x$. The last statement follows from Lemma 17 and Fact 9.

Lemma 22. Let $X \subseteq G^{\prime}$ be a $G$-normal subgroup of $G^{\prime}$. Then there are finitely many local elements $x_{1}, \ldots, x_{u}$ for which $X=R x_{1}+\cdots+R x_{u}$.

Proof. We prove by induction that $X \cap S_{i}$ satisfies this property. For $i=1$ the result follows from Lemma 3 (minimal elements of $S_{1}$ are local elements of $S_{1}$ also). Similarly, $X \cap S_{i} / X \cap S_{i-1}=\left(R x_{1}+\cdots+R x_{u}\right) / X \cap S_{i-1}$ for some minimal elements of $S_{i}$. By Lemma 20, we may choose the elements $x_{i}$ to be local elements. Now the lemma follows by induction.

The next lemma is a variant of Nakayama's lemma (see, e.g., [McD]).

Lemma 23. Let $x \in G^{\prime}$ be a local element. Then

$$
M_{x}\left(S_{i} \cap R x\right) \subseteq S_{i-1}
$$

for all $i$. Also, for any minimal element $y \in R x, M_{x}=M_{y}$. Thus any minimal element of $R x$ is also a local element.

Proof. Let $y \in R x$ be a minimal element. By Fact 9 and Lemma 17 for some integer $n, M_{x}^{n} \subseteq$ ann $x \subseteq$ ann $y \subseteq M_{y}$. But $M_{y}$ is maximal, thus prime, so $M_{x}=M_{y}$. This proves the second claim. The third claim follows.

Let $y \in S_{i} \cap R x$. Say $y=r_{0} x$. If $y \in S_{i-1}$ then $M_{x} y \subseteq S_{i-1}$. Assume therefore that $y \in S_{i} \backslash S_{i-1}$. Consider the ideal

$$
M_{y}=\left\{r \in R: r(y) \in S_{i-1}\right\} .
$$

Since $y \notin S_{i-1}, M_{y} \neq R$. Since ann $x \subseteq M_{y}$ and $R /$ ann $x$ is a local ring, by Lemma 21 we have $M_{y} \subseteq M_{x}$. Conversely, let $r \in M_{x}$. Assume $r(y) \in S_{i}$. Since $0 \neq\left(R y+S_{i-1}\right) / S_{i-1}$ is $G / S_{i-1}$-minimal (because $R y \subseteq R x$ and $x$ is minimal), for some $s \in R$

$$
\operatorname{sr}(y) \equiv y\left(S_{i-1}\right) .
$$

But $r \in M_{x}$, so $r^{n} \in \operatorname{ann}(x)$ for some natural number $n$. Therefore

$$
r^{n}(y)=r^{n} r_{0}(x)=r_{0} r^{n}(x)=0 .
$$

But also

$$
s^{n} r^{n}(y) \equiv y\left(S_{i-1}\right) \text {. }
$$

Thus $y \in S_{i-1}$, a contradiction. This lemma is proved.

Corollary 24. Let $x, y \in G^{\prime}$ be two local elements. If $R x \cap R y \neq 0$ then $M_{x}=M_{y}$. 
Proof. Let $z \in R x \cap R y \backslash\{0\}$ be a minimal element. Assume $z \in S_{i} \backslash S_{i-1}$. If $M_{x} \neq M_{y}$, then $R=M_{x}+M_{y}$. Thus

$$
1=\alpha+\beta
$$

for some $\alpha \in M_{x}, \beta \in M_{y}$. So

$$
z=\alpha(z)+\beta(z) .
$$

But by Lemma $23 \alpha(z), \beta(z) \in S_{i-1}$. Hence $z \in S_{i-1}$, a contradiction.

Lemma 25. Let $x, y \in G^{\prime}$ be two local elements. Then the following are equivalent:

(i) ann $x \subseteq M_{y}$;

(ii) $M_{x}=M_{y}$;

(iii) ann $y \subseteq M_{x}$.

Proof. It is enough to show that (i) $\Leftrightarrow$ (ii). (i) $\Rightarrow$ (ii). Since $M_{x} / \operatorname{ann} x$ is a nilpotent ideal $M_{x}^{n} \subseteq$ ann $x \subseteq M_{y}$ for some $n$. But $M_{y}$ is a prime ideal. Thus $M_{x}^{n} \subseteq M_{y}$ implies $M_{x} \subseteq M_{y}$. Since they are maximal $M_{x}=M_{y}$.

(ii) $\Rightarrow$ (i). $\operatorname{ann}(x) \subseteq M_{x}=M_{y}$.

Now we define an equivalence relation on the set of local elements of $G^{\prime}$ (which is a definable subset, but we do not need this): If $x, y \in G^{\prime}$ are local, we say that $x$ is equivalent to $y$, and we write $x \sim y$, if ann $x \subseteq M_{y}$. By Lemma 25 this is an equivalence relation. By Lemma $22, G^{\prime}=R x_{1}+\cdots+R x_{u}$ for some local elements $x_{1}, \ldots, x_{u}$. Let us fix these elements once for all and restrict our equivalence relation to the set $\left\{x_{1}, \ldots, x_{u}\right\}$.

Let

$$
R_{j}=\bigcap_{x_{i} \ngtr x_{j}} \operatorname{ann} x_{i} .
$$

Let us assume, for the sake of the simplicity of notation and without loss of generality, that $x_{1}, \ldots, x_{l}$ are unique representatives of each equivalence class. Each $R_{j}$ is an ideal of $R$. Since we work modulo ann $G^{\prime}, R_{j} \cap R_{k}=0$ if $j \neq k$. We will denote $M_{x_{j}}$ by $M_{j}$.

Proposition 26. $R=\bigoplus_{j=1}^{l} R_{j}$.

Proof. Clearly $R_{j} R_{k}=0$ if $j \neq k$. Also, if $x_{j} \not x_{k}, R=M_{j}+\operatorname{ann} x_{k}$. Thus

$$
1=\alpha_{j k}+\beta_{j k} \quad(j \neq k)
$$

for $\alpha_{j k} \in M_{j}, \beta_{j k} \in \operatorname{ann}\left(x_{k}\right)$. Let us first fix $j$ and vary $x_{k}$ over the complement of the equivalence class of $x_{j}$. Multiply them:

$$
1=\prod_{x_{k} \not x_{j}}\left(\alpha_{j k}+\beta_{j k}\right)=\gamma_{j}+\delta_{j}
$$


where $\gamma_{j} \in M_{j}, \delta_{j}=\prod_{x_{k} \nsucc x_{j}} \beta_{j k} \in R_{j}$. Now vary $j$ over the set of equivalence classes and multiply then:

$$
1=\prod_{j=1}^{l}\left(\gamma_{j}+\delta_{j}\right)=\eta+\eta_{1}+\cdots+\eta_{l}
$$

where $\eta=\gamma_{1} \cdots \gamma_{l} \in M_{1} \cap \cdots \cap M_{l}$ and $\eta_{j} \in R_{j}$. Since each $M_{i} /$ ann $x_{j}$ is nilpotent if $x_{i} \sim x_{j}$, some power of $M_{1} \cap \cdots \cap M_{l}$ is the zero ideal. Let $n$ be this power. Then

$$
1=\left(\eta+\eta_{1}+\cdots+\eta_{l}\right)^{n}=\eta^{n}+\varphi_{1}+\cdots+\varphi_{n}=\varphi_{1}+\cdots+\varphi_{n}
$$

where $\varphi_{j} \in R_{j}$. Thus $R=\sum R_{j}$. Since $R_{j} \cap \sum_{j \neq k} R_{k}=0$ we have

$$
R=\bigoplus_{j=1}^{l} R_{j} .
$$

Lemma 27. $G^{\prime}=\bigoplus_{j=1}^{l} R_{j} G^{\prime}$.

Proof. Since $R=\bigoplus_{j=1}^{l} R_{j}$, we have $G^{\prime}=\sum_{j=1}^{l} R_{j} G^{\prime}$. Write $1=e_{1}+\cdots+e_{l}$. with $e_{j} \in R_{j}$. Since $R_{j} R_{k}=0$ if $j \neq k$ we have $e_{i} e_{j}=0$ if $i \neq j$ and $e_{i}^{2}=1$. Let us show that they direct-sum: if

$$
\beta_{k} x_{k} \in R_{k} G^{\prime} \cap \sum_{j \neq k} R_{j} G^{\prime} \quad\left(\beta_{k} \in R_{k}\right),
$$

multiplying both sides by $1=e_{1}+\cdots+e_{l}$, we get

$$
\beta_{k} x_{k}=0 \text {. }
$$

This proves the lemma.

Notice that by Corollary $19, R_{j} G^{\prime}$ is definable. But we do not yet know if $R_{j}$ is interpretable in $G$.

Lemma 28. If $x, y \in R_{j} G^{\prime}$ are minimal elements then $M_{x}=M_{y}$ (i.e., $x \sim y$ ). Proof. Since for the (fixed) local elements $x_{1}, \ldots, x_{u}$ we have

$$
G^{\prime}=R x_{1}+\cdots+R x_{u}
$$

and since $R_{j} x_{i}=0$ if $x_{i} \not x_{j}$ we have

$$
R_{j} G^{\prime}=R_{j} x_{j 1}+\cdots+R_{j} x_{j t}
$$

for some $x_{j 1}, \ldots, x_{j t} \in\left\{x_{1}, \ldots, x_{u}\right\}$ for which $M_{x_{j 1}}=\cdots=M_{x_{j 1}}=M_{j}$. For each $i$ some power of $M_{j}$ is in ann $x_{j i}$. Thus for some integer $n$

$$
M_{j}^{n} \subseteq \bigcap_{i=1}^{t} \operatorname{ann} x_{j i}=\operatorname{ann} R_{j} G^{\prime} .
$$


Now if $z \in R_{j} G^{\prime}$ then ann $R_{j} G^{\prime} \subseteq M_{z}$. Thus

$$
M_{j}^{n} \subseteq M_{z} \text {. }
$$

If $z$ is minimal then $M_{z}$ is a prime ideal; thus the above inclusion gives

$$
M_{j} \subseteq M_{z} .
$$

But $M_{j}$ is also a maximal ideal, thus $M_{j}=M_{z}$.

To prove that $R$ is interpretable, in view of Proposition 26 and Lemma 27 we need only show that the action of $R_{j}$ on $R_{j} G^{\prime}$ is interpretable (this is saying that $R_{j}$ is interpretable). Thus we may assume for a while that $R=R_{j}$, $G^{\prime}=R_{j} G^{\prime}$. With these hypotheses we have the following property: if $x, y \in G^{\prime}$ are minimal then $M_{x}=M_{y}$. We may therefore write $M$ for $M_{x}$. Clearly if $x \in G^{\prime}$, then ann $x \subseteq M$.

Lemma 29 (with the above additional hypothesis). $R$ is a local ring with $M$ as the maximal ideal. Furthermore, $M$ is nilpotent.

Proof. Let $G^{\prime}=R x_{1}+\cdots+R x_{u}, x_{i} \in G^{\prime}$ local, $r \in R \backslash M$. Then for each $i=1, \ldots, u$ there is an $s_{i} \in R$ for which

$$
r s_{i} \equiv 1 \bmod \left(\operatorname{ann} x_{i}\right)
$$

(because $R /$ ann $x_{i}$ is a local ring with $M /$ ann $x_{i}$ as its maximal ideal). Thus

$$
\prod_{i=1}^{u}\left(1-r s_{i}\right) \in \bigcap_{i=1}^{u} \operatorname{ann} x_{i}=\operatorname{ann} G^{\prime}=0 \text {. }
$$

The left-hand side is of the form $1-r t$. Thus $r t=1$ and $r$ is invertible.

Let us now show that $M$ is nilpotent. Since $M /$ ann $x_{i}$ is nilpotent, for some integer $n_{i}, M^{n_{i}} \subseteq$ ann $x_{i}$. Thus

$$
M^{n_{1} \cdots n_{u}} \subseteq \bigcap_{i=1}^{u} \operatorname{ann} x_{i}=0 .
$$

To prove that $R$ is interpretable we need some more notation. Assume that $G^{\prime}=S_{l}(l \in \mathbf{N})$. Then we know by Lemma 23 that $M G^{\prime} \subseteq S_{l-1}$. Define

$$
M(i)=\left\{r \in R: r G^{\prime} \subseteq S_{l-i}\right\} .
$$

$M(i)$ is an ideal of $R . M(i) \supseteq M(i+1), M=M(1), M^{i} \subseteq M(i)$ (by Lemma 23 again). Since $M S_{l-i} \subseteq S_{l-i-1}$ (Lemmas 22 and 23) we have

$$
M . M(i) \subseteq M(i+1) .
$$

This shows that $M(i) / M(i+1)$ is a vector space over $R / M$. We would like to show that it is finite dimensional. Since $R /$ ann $x_{j}$ is an interpretable ring (Lemma 17), it is $\omega$-stable; thus

$$
\left(M(i)+\operatorname{ann} x_{j} / \operatorname{ann} x_{j}\right) /\left(M(i+1)+\operatorname{ann} x_{j} / \operatorname{ann} x_{j}\right)
$$

is a finite-dimensional vector space over $\left(R /\right.$ ann $\left.x_{j}\right) /\left(M\right.$ ann $\left.x_{j}\right)$. Let us record this in a nicer form. 
Lemma 30 (hypothesis as in Lemma 29). $M(i) /(M(i+1)+(M(i) \cap \operatorname{ann} x))$ is a finite-dimensional vector space over $R / M$ for any $x \in G^{\prime}$.

Proof.

$$
\begin{aligned}
(M(i) & +\operatorname{ann} x / \operatorname{ann} x) /(M(i+1)+\operatorname{ann} x / \operatorname{ann} x) \\
& \simeq M(i)+\operatorname{ann} x / M(i+1)+\operatorname{ann} x \\
& =M(i)+M(i+1)+\operatorname{ann} x / M(i+1)+\operatorname{ann} x \\
& \simeq M(i) / M(i) \cap(M(i+1)+\operatorname{ann} x) \\
& =M(i) / M(i+1)+(M(i) \cap \operatorname{ann} x)
\end{aligned}
$$

with natural isomorphisms. Also

$$
(R / \operatorname{ann} x) /(M / \operatorname{ann} x) \simeq R / M,
$$

again with natural isomorphism. The lemma follows from the previous remarks.

Lemma 31 (hypothesis as in Lemma 29). $M(i) / M(i+1)$ is a finite-dimensional vector space over $R / M$.

Proof. Let $G^{\prime}=R x_{1}+\cdots+R x_{u}$ be as usual. We first show that

$$
\bigcap_{j=1}^{u}\left(M(i+1)+\left(M(i) \cap \operatorname{ann} x_{j}\right)\right)=M(i+1) .
$$

Let $r$ be in the left-hand side. Thus $r=\alpha_{j}+\beta_{j}$ where $\alpha_{j} \in M(i+1)$, $\beta_{j} \in$ ann $x_{j}$. Thus

$$
r\left(x_{j}\right)=\alpha_{j}\left(x_{j}\right) .
$$

But $\alpha_{j} \in M(i+1)$, so $r\left(x_{j}\right) \in S_{l-(i+1)}$. This is true for all $j$. Hence $r\left(G^{\prime}\right) \subset$ $S_{l-(i+1)}$, so $r \in M(i+1)$. This proves the claim.

Next we consider the product of natural maps:

$$
M(i) / M(i+1) \rightarrow \prod_{j=1}^{u} M(i) /\left(M(i+1)+\left(M(i) \cap \operatorname{ann} x_{j}\right)\right) .
$$

By the above claim, this $R / M$-vector space homomorphism is $1-1$. Since by the previous lemma each component of the right-hand side is a finite-dimensional vector space over $R / M$, the lemma is proved.

Lemma 32 (hypothesis as in Lemma 29). $R$ is an interpretable ring.

Proof. Choose a base $\bar{x}_{i 1}, \ldots, \bar{x}_{i t_{i}}$ of $M(i) / M(i+1)$ over $R / M$. Let $x_{i 1}, \ldots$, $x_{i t_{i}}$ be a set of representatives in $M(i)$. Let $r \in R$. By Zil'ber $R / M$ is interpretable: there is an $s \in \mathbf{Z}[T]$ of bounded length such that $r-s \in M$. Now each element of $M$ can be written as linear combinations of $x_{i j}$ 's with coefficients $r_{i j} \in R$ of bounded length. The lemma follows. 
In Lemma 32, we proved more:

Lemma 33 (hypothesis as in Lemma 29). There is a fixed integer $k$ such that if $r \in R$ then there is an element $\gamma \in \mathbf{Z}[T]$ of length $\leq k$ for which

$$
r=\gamma \in \mathbf{Z}[T] / \text { ann } G^{\prime} \text {. }
$$

We do not need the hypothesis of Lemma 29 anymore:

Theorem 3. $R$ is interpretable in $G$ (its interpretation is given in Lemma 33). The full group $\widetilde{G}=G^{\prime} \rtimes R^{*}$ is a connected solvable group of class 2 and of finite Morley rank.

Proof. With the notation of Proposition 26, Lemma 33 says that $R_{j}$ is interpretable. Hence $R$ is interpretable. Thus $\widetilde{G}$ is a solvable group of class 2 which has finite Morley rank. To show that it is connected we need to show that $R$ is. We leave this to the next theorem.

Theorem 4. Let $G$ be a connected centerless solvable group of class 2 and of finite Morley rank. Let $\widetilde{G}$ be the full group of $G$. Then $\widetilde{G} \simeq \bigoplus_{i=1}^{l}\left(G_{i}^{\prime} \rtimes R_{i}^{*}\right)$ where

(i) $R_{i}$ is an interpretable connected local ring, with nilpotent maximal ideals. $G_{i}^{\prime}<G^{\prime}$ and $G_{i}^{\prime} \triangleleft G$. Furthermore, if $i \neq j$ then $R_{i}^{*}$ acts as identity on $G_{j}^{\prime}$.

(ii) $R_{i}$ is commutative, has an identity element, and every ideal is definable and connected.

(iii) $R_{i}$ is Artinian and Noetherian.

(iv) $\widetilde{G}^{\prime}=\bigoplus G_{j}^{\prime}=G^{\prime}$.

Proof. At this point we may use Cherlin-Reineke (Fact 9) but we have all the information in our possession already. Let $G_{i}^{\prime}$ be $R_{i} G^{\prime}$; then $G^{\prime}=\bigoplus G_{i}^{\prime}$. We know that $R=\bigoplus R_{i}$. Thus $\widetilde{G}=G^{\prime} \rtimes R^{*}=\left(\bigoplus G_{i}^{\prime}\right) \rtimes\left(\bigoplus R_{i}^{*}\right) \simeq \bigoplus\left(G_{i}^{\prime} \rtimes R_{i}^{*}\right)$.

By Lemma 32, $R_{i}$ is interpretable. $R_{i}$ is connected because $R_{i} / M_{i}$ is an algebraically closed field (so is connected) and $M_{i}^{j} / M_{i}^{j+1}$ are vector spaces over $R_{i} / M_{i}$ (so are connected). This proves (i).

Let $I \triangleleft R_{i}$. Since $R_{i}$ has an identity element

$$
I=R_{i} I=\left\langle R_{i} a: a \in I\right\rangle .
$$

Since $R_{i} a \simeq R_{i} /$ ann $a, R_{i} a$ is connected. by Zil'ber, $I$ is definable and connected. This proves (ii). (iii) follows from (ii).

Notice that all fields found by Zil'ber's method in $G_{i}^{\prime} \rtimes R_{i}^{*}$ is isomorphic to $R_{i} / M_{i}$. Notice also that $R_{i}$ acts on $S_{j} / S_{j-1}$ by scalar multiplication, where $S_{j}$ is the $j$ th socle of $G_{i}^{\prime}$.

Kathryn Enochs and the author have recently shown [E-N] that $G_{i}^{\prime} \rtimes R_{i}^{*}$ is an algebraic group over the field $R_{i} / M_{i}$.

We can find a smaller imbedding of $G$. 
Theorem 5. Let $G$ be a connected centerless solvable group of class 2 and finite Morley rank. Then there are interpretable abelian subgroups $T_{i}$ acting on $G^{\prime}$; there are finitely many, definable G-normal subgroups $G_{i}^{\prime}<G^{\prime}$ such that

(i) $G \subseteq \bigoplus_{i=1}^{m}\left(G_{i}^{\prime} \rtimes T_{i}\right)$.

(ii) $\mathrm{Z}\left[T_{i}\right] /$ ann $G_{i}^{\prime}$ is an interpretable, local, Noetherian ring with nilpotent maximal ideals and algebraically closed residue fields.

(iii) $T_{i}$ is a homomorphic image of $T$ where $T$ is a definable complement of $G^{\prime}$ in $G$.

Proof. Let $R$ be as before. Let $e_{1}, \ldots, e_{m}$ be atomic idempotents of $R$. (Then $R_{i}=R e_{i}$ with the above notation.) Set $T_{i}=T e_{i}, G_{i}^{\prime}=R_{i} G^{\prime}$.

Remark. Most of the results of this section also hold for modules (with two sorted languages $R$ and $M$ ) over commutative rings.

\section{FITTING SUBGROUP}

Let $G$ be a connected solvable group of finite Morley rank. By Facts 4 and 5, $G^{\prime}$ is connected and nilpotent. Let $F$ be a nilpotent, normal, connected subgroup of $G$ which is maximal for these properties. If $F_{1}$ and $F_{2}$ are two such groups, then $\left\langle F_{1}, F_{2}\right\rangle=F_{1} F_{2}$ is nilpotent and connected. Thus $F_{1}=F_{2}$. Therefore such a subgroup is unique. We call it the Fitting subgroup of $G$. It contains $G^{\prime}$.

If $H$ is a nilpotent, definable, normal subgroup of $G$ then $H^{0} \subseteq F$. Thus $F H / F$ is finite.

If $G$ is algebraic (say over $K), G / F$ is a quotient of a torus $\left(\simeq\left(K^{*}\right)^{n}\right.$, some $n$ ). Thus in this case $G / F$ is divisible. We generalize this.

Theorem 6. Let $G$ be a solvable connected group of finite Morley rank, $F$ its Fitting subgroup. Then $G / F$ is divisible.

Proof. $G / F$ is abelian (because $G^{\prime} \subseteq F$ ). Thus by Fact 7 ,

$$
G / F=B / F \oplus D / F
$$

where $D / F$ is divisible and $B / F$ has bounded exponent. If $n$ is this exponent, then

$$
D=\left\{g^{n}: g \in G\right\} F .
$$

Thus $D$ is definable. Since $D / F$ has finite Morley rank, for any fixed integer $m, D / F$ has only finitely many elements of order $m$ (if not we can find infinitely many copies of $\mathbf{Z}_{p^{\infty}}$ for some prime number $p$ ).

$B$ may not be definable, but the set

$$
C=\left\{g \in G: g^{n} \in F\right\}
$$

is definable and contains $B$. Since $G / F$ is abelian, $C$ is a subgroup of $G$. It is clearly normal. By the above discussion, $C \cap D$ is finite, thus $[C: B]<\infty$. Notice also that $F \subseteq C^{0}$. 
We will show that $C^{0}$ is nilpotent. Since $C^{\prime} \subseteq F$, we need only show that

$$
\underbrace{\left[C^{0},\left[C^{0}, \ldots,\left[C^{0}, F\right] \cdots\right]\right]}_{m \text { times }}=1
$$

for some $m$.

By considering socles and centers we can find a sequence of definable normal subgroups $\left(F_{i}\right)_{i}$ :

$$
0=F_{0} \varsubsetneqq F_{1} \varsubsetneqq F_{2} \varsubsetneqq \cdots \varsubsetneqq F_{n}=F
$$

in such a way that either $F_{i} / F_{i-1}$ is $G / F_{i-1}$-minimal and $Z\left(G / F_{i-1}\right) \cap F_{i} / F_{i-1}=$ 1 or

$$
F_{i} / F_{i-1}=Z\left(G / F_{i-1}\right) \text {. }
$$

We want to show that $\left[C^{0}, F_{i}\right] \subseteq F_{i-1}$ for all $i$. This will clearly imply that $C^{0}$ is nilpotent.

If $F_{i} / F_{i-1}=Z\left(G / F_{i-1}\right)$ then $\left[G, F_{i}\right] \subseteq F_{i-1}$ by definition of $Z\left(G / F_{i-1}\right)$.

If not, the action of $C$ on $F_{i} / F_{i-1}$ is a vector-space action; i.e., $F_{i} / F_{i-1}$ is a 1-dimensional vector space over an algebraically closed field and $C$ acts on it as it should. Since elements of $C$ have the same finite-order modulo $F$, these actions are automorphisms of order $n$. Thus we have finitely many possible actions. Therefore $C^{0}$ acts trivially (i.e. as identity) on $F_{i} / F_{i-1}$; in other words, $\left[F_{i}, C^{0}\right] \subseteq F_{i-1}$.

Thus $C^{0}$ is nilpotent. So $C^{0} \subseteq F \subseteq C^{0}$. Thus $C^{0}=F$.

Since $F \subseteq B$ and $|C / B|$ is finite, this shows that $B / F$ is finite. But $G / F$ is connected. So $B / F=1$ and $G / F=D / F$ is divisible.

Corollary. Given any integer $n, G / F$ has only finitely many elements of order $n$.

Recently, the author and Kathryn Enochs proved that in case $G^{\prime}$ is abelian and $G$ is centerless, then $F=C_{G}(S)^{0}$. The proof relies heavily on the methods, results, and classification of local rings of the previous section. It would be interesting to find a simpler proof of this fact. We do not know if the statement $F=C_{G}\left(S_{i}\right)^{0}$ (for some $i$ ) is true without any solvability class assumption.

Conjecture. Let $G$ be a connected solvable group of finite Morley rank. Let $F$ be the Fitting subgroup of $G$. Then $F$ splits (not necessarily definably) in $G$.

The reason to believe in this conjecture will be more apparent in the next section.

The conjecture has recently been proved by the author and Kathryn Enochs (see [E-N]) in case $G$ is centerless and solvable of class 2 .

\section{LiNEAR ALGEBRAIC GROUPS}

Let $G$ be a linear algebraic group over an algebraically closed field $K$. If $G$ is algebraically connected, we say that $G$ is a-connected. If $G$ is model theoretically connected, we say that $G$ is $m$-connected. 
The following theorem was proved with Anand Pillay.

Theorem 7. Let $G$ be a centerless a-connected solvable linear algebraic group. Then the unipotent subgroup $\mathbf{U}$ is definable and if the language is the pure groups language $\mathbf{U}$ is also m-connected.

Proof. We define the following subgroups:

$T=$ a maximal torus of $G$.

$\overline{\mathbf{U}}=$ definable closure of $\mathbf{U}$,

$\overline{\overline{\mathbf{U}}}=$ Zariski closure of $\overline{\mathbf{U}}$,

$\overline{\overline{\mathbf{U}}}^{0}=a$-connected component of $\overline{\overline{\mathbf{U}}}$.

By Fact $8, \overline{\mathbf{U}}$ is nilpotent. It is known that $\overline{\overline{\mathbf{U}}}$ is nilpotent (see Borel [Bo, $\S 2.4$, Corollary]). Then $\overline{\overline{\mathbf{U}}}^{0} \cap T$ is central in $\overline{\overline{\mathbf{U}}}^{0}$ (see [Sp, Corollary 6.8(i), p. 145]). Thus $\overline{\mathbf{U}}^{0} \cap T$ commutes with $\mathbf{U}$ (because $\mathbf{U} \subseteq \overline{\overline{\mathbf{U}}}^{0}$ ). Since $T$ is abelian, $\overline{\overline{\mathbf{U}}}^{0} \cap T$ also commutes with $T$. Thus $\overline{\overline{\mathbf{U}}}^{0} \cap T$ commutes with $G$ $(=\mathbf{U} \rtimes T)$. Thus $\overline{\mathbf{U}}^{0} \cap T=1$. Since $\mathbf{U} \subseteq \overline{\mathbf{U}}^{0}$, this implies that $\mathbf{U}=\overline{\overline{\mathbf{U}}}^{0}$. Thus $[\overline{\overline{\mathbf{U}}}: \mathbf{U}]<\infty$. Since $\mathbf{U} \subseteq \overline{\mathbf{U}} \subseteq \overline{\overline{\mathbf{U}}}$, we also have $[\overline{\mathbf{U}}: \mathbf{U}]<\infty$. Thus $\overline{\mathbf{U}}=\mathbf{U} \rtimes(\overline{\mathbf{U}} \cap T)$ with $|\overline{\mathbf{U}} \cap T|<\infty$.

Let $q$ be the order of $\overline{\mathbf{U}} \cap T$. Let $p$ be the characteristic of the field. Since $T$ is isomorphic to $\left(K^{*}\right)^{n}$ we have $(p, q)=1$. Let $u t \in \overline{\mathbf{U}}=\mathbf{U} \rtimes(\overline{\mathbf{U}} \cap T)$. Then

$$
(u t)^{q}=v t^{q}=v
$$

for some $v \in \mathbf{U}$. Thus

$$
\overline{\mathbf{U}}^{q} \subseteq \mathbf{U} .
$$

On the other hand, since $(p, q)=1$ we have

$$
\mathbf{U}=\mathbf{U}^{q} \subseteq \overline{\mathbf{U}}^{q},
$$

thus

$$
\mathbf{U}=\overline{\mathbf{U}}^{q}
$$

and $\mathbf{U}$ is definable.

We now assume that the language is pure group language. Let $\mathbf{U}^{0}$ be the $m$ connected component of $\mathbf{U} . \mathbf{U}^{0}$ is a constructible set in the Zariski topology of $G$ (i.e., finite union of intersections of an open and closed set). It contains an open in its closure [Po2]. Thus $\mathbf{U}^{0}$ is an open subgroup of $\mathbf{U}$. Thus it is also closed, hence $\mathbf{U}=\mathbf{U}^{0}$.

Fitting subgroup has the meaning we gave to it in $\S 7$.

Corollary ( $G$ and $\mathbf{U}$ as above and in the pure language of groups). $\mathbf{U}$ is the Fitting subgroup of $G$.

Proof. Let $F$ be the Fitting subgroup of $G$. By Theorem 7, $\mathbf{U} \subseteq F$. Replacing $\mathbf{U}$ by $F$ in the first part of the proof of Theorem 7, we see that $F=\mathbf{U}$. 
Question. Does the last statement of Theorem 7 hold if the language is richer (but $G$ is still $\omega$-stable)?

\section{MiNimal NONNILPOTENT GROUPS}

Cherlin proved in [Ch] that a centerless connected group $G$ of Morley rank 2 is an affine group over an algebraically closed field, i.e.,

$$
G \simeq\left\{\left(\begin{array}{ll}
1 & a \\
0 & b
\end{array}\right): a \in K, b \in K^{*}\right\}=K \rtimes K^{*}
$$

for some algebraically closed field $K$. In this section we generalize this result.

Theorem 8. Let $G$ be a connected centerless solvable group of finite Morley rank. Assume all definable proper connected subgroups of $G$ are nilpotent. Then for some algebraically closed field $K$ and some definable $T<K^{*}$ for which $T+$ $\cdots+T=K$ (finite number) we have $G=K \rtimes T$ with $T$ acting on $K$ by field multiplications.

Proof. Let $S$ be the socle of $G, g \in G$ be as in Lemma 5. Let $X$ be as in Lemma 7:

$$
X=\left\{x \in G^{\prime}:[G, x] \subseteq S\right\} .
$$

By Lemma 7 ,

$$
X=S \oplus C_{x}(g) .
$$

Assume $C_{x}(g) \neq 1$. Let $x \in C_{x}(g)-\{1\}$. Then $g^{m} \in C_{G}(x)^{0}$ for some integer $m$. Replacing $g$ by $g^{m}$, we may suppose that $g \in C_{G}(x)^{0}$. By hypothesis $C_{G}(x)^{0} \neq G$, so $C_{G}(x)^{0}$ is nilpotent. But $S \subseteq C_{G}(x)^{0}, g \in C_{G}(x)^{0}$, thus $[g[g, \ldots,[g, S] \cdots]]=1$; this is a contradiction because $C_{s}(g)=1$. Thus $X=S$. This means that

$$
G^{\prime} / S \cap Z(G / S)=1 .
$$

In particular, $G / S$ is not nilpotent. Thus $G / S$ satisfies the hypothesis of our statement. Thus by induction $G / S=G^{\prime} / S \rtimes T / S$. By Proposition 11, $G=G^{\prime} \rtimes$ $T_{1}$ for some definable $T_{1}$ ( $T$ is definable by induction hypothesis). $S \rtimes T_{1} \subseteq G$ and $S \rtimes T_{1}$ is not nilpotent. Thus $G=S \rtimes T_{1}$. If $A_{1} \subseteq S$ is a minimal normal then $G=A_{1} \rtimes T_{1}$ for the same reason. Since $G$ is centerless we get the result by Zil'ber.

The natural question is now:

Question. Suppose $G$ is a centerless connected group of finite Morley rank. Assume every proper definable connected subgroup of $G$ has a nontrivial center. What can we say about $G$ ?

\section{REFERENCES}

[B.Ch.Mac] W. Baur, G. Cherlin and A. Macintyre, Totally categorical groups and rings, J. Algebra 57 (1979), 407-440.

[Bo] A. Borel, Linear algebraic groups, Benjamin, New York, 1969. 
[Ch] G. Cherlin, Groups of small Morley rank, Ann. Math. Logic 17 (1979), 1-28.

[Ch-R] G. Cherlin and J. Reineke, Stability and categoricity of commutative rings, Ann. Math. Logic 10 (1979), 376-399.

[Co] I. S. Cohen, On the structure and ideal theory of complete local rings, Trans. Amer. Math. Soc. 59 (1946), 54-104.

[E-N] E. Enochs and A. Nesin, On 2-step solvable groups of finite Morley rank, Proc. Amer. Math. Soc. (to appear).

[La] D. Lascar, Stabilité en théorie des modèles, Publ. Inst. Math. Pure Appl. Univ. Catholique de Louvain (1986). (French)

[Ma] D. Marker, Expansions of C, preprint, 1988.

[Mac1] A. Macintyre, On $\omega_{1}$-categorical theories of abelian groups, Fund. Math. 70 (1971), 253270.

[Mac2] _ On $\omega_{1}$-categorical theories of fields, Fund. Math. 71 (1971), 1-25.

[McD] B. R. McDonald, Geometric algebra over local fields, Marcel Dekker, New York, 1976.

[Mo] M. D. Morley, Categoricity in power, Trans. Amer. Math. Soc. 114 (1965), 514-538.

[Ne1] A. Nesin, A survey of results in groups of finite Morley rank, preprint, 1985.

[Ne2] _ - Solvable groups of finite Morley rank, J. Algebra 121 (1989), 26-39.

[Pi] A. Pillay, An introduction to stability theory, Oxford Logic Guides 8 (1983).

[Po1] B. Poizat, Cours de théories des modèles, Nur Al-Mantiq Wal-Ma'rifah Villeurbanne, 1985. (French)

[Po2] _ Groupes stables, Nur Al-Mantiq Wal-Ma'rifah, 1987. (French)

[Sa] P. Samuel, Algèbre locale, Paris, 1953. (French)

[Sp] T. A. Springer, Linear algebraic groups, Birkhäuser, Basel, 1981.

[Ta] K. Tanaka, Some structure theorems for $\omega$-stable groups, Proc. Japan Acad. Ser. A Math. Sci. 63 (1987).

[Th1] S. Thomas, Groups of finite Morley rank, handwritten notes.

[Th2] _ The classification of simple periodic linear groups, Arch. Math. (Basel) 41 (1983), 103-116.

[Zi1] B. Zil'ber, Groups with categorical theories, Abstract of papers presented at the Fourth AllUnion Symposium on Group Theory, Novosibirsk, 1973., Math. Inst. Sibirsk. Adel. Akad. Nauk SSSR, pp. 63-68. (Russian)

[Zi2] _ - The structure of models of categorical theories and the non-finite axiomatizability problem, preprint, mimeographed by VINITI, Dep. N2800-77 (Kemerovo, SSSR, 1977). (Russian)

[Zi3] _ Some model theory of simple algebraic groups over algebraically closed fields, Colloq. Math. 48 (1984), 173-180.

Department of Mathematics, University of Notre Dame, Notre Dame, Indiana 46556

Current address: Department of Mathematics, University of California, Irvine, California 92717 